\title{
On the completeness of complete markets
}

Citation for published version (APA):

Herings, P. J. J., \& Rohde, K. I. M. (2004). On the completeness of complete markets. METEOR, Maastricht University School of Business and Economics. METEOR Research Memorandum No. 051 https://doi.org/10.26481/umamet.2004051

Document status and date:

Published: 01/01/2004

DOI:

10.26481/umamet.2004051

Document Version:

Publisher's PDF, also known as Version of record

\section{Please check the document version of this publication:}

- A submitted manuscript is the version of the article upon submission and before peer-review. There can be important differences between the submitted version and the official published version of record.

People interested in the research are advised to contact the author for the final version of the publication, or visit the DOI to the publisher's website.

- The final author version and the galley proof are versions of the publication after peer review.

- The final published version features the final layout of the paper including the volume, issue and page numbers.

Link to publication

\footnotetext{
General rights rights.

- You may freely distribute the URL identifying the publication in the public portal. please follow below link for the End User Agreement:

www.umlib.nl/taverne-license

Take down policy

If you believe that this document breaches copyright please contact us at:

repository@maastrichtuniversity.nl

providing details and we will investigate your claim.
}

Copyright and moral rights for the publications made accessible in the public portal are retained by the authors and/or other copyright owners and it is a condition of accessing publications that users recognise and abide by the legal requirements associated with these

- Users may download and print one copy of any publication from the public portal for the purpose of private study or research.

- You may not further distribute the material or use it for any profit-making activity or commercial gain

If the publication is distributed under the terms of Article $25 \mathrm{fa}$ of the Dutch Copyright Act, indicated by the "Taverne" license above, 


\title{
On the Completeness of Complete Markets.
}

\author{
P. Jean-Jacques Herings ${ }^{1} \quad$ Kirsten I.M. Rohde ${ }^{2}$
}

December 20, 2004

\footnotetext{
${ }^{1}$ Department of Economics, Maastricht University, P.O. Box 616, 6200 MD Maastricht, The Netherlands. E-mail: P.Herings@algec.unimaas.nl. The author would like to thank the Netherlands Organisation for Scientific Research (NWO) for financial support.

${ }^{2}$ Department of Economics, Maastricht University, P.O. Box 616, 6200 MD Maastricht, The Netherlands. E-mail: K.Rohde@algec.unimaas.nl
} 


\begin{abstract}
We reconsider the allocational invariance of equilibria to different formulations of market completeness. We identify the so-far neglected assumption of sophisticated behavior as crucial to this result. The paper studies three market structures. First, the Arrow-Debreu setting is considered. Second, sequentially complete markets are analyzed, where goods on the spot markets and all contingent one-period ahead commodities can be traded in every state. Finally, complete markets are analyzed, where all possible contingent commodities can be traded at every state. Preferences may be time-consistent or time-inconsistent. A distinction is made between naïve and sophisticated behavior. For economies with timeinconsistent preferences, Arrow-Debreu equilibria are not related to either sequentially complete equilibria or complete equilibria. It does hold that every equilibrium consumption that can be attained in sequentially complete markets, can also be attained in complete markets. An example shows that the converse is not true for naïve economies. Finally, when preferences are restricted to be time-consistent and households are sophisticated, the three market structures yield the same equilibrium consumption. Surprisingly, for naïve households, this result is not true, even when preferences are time-consistent.
\end{abstract}

Keywords: Time-consistent preferences, Time-inconsistency, Competitive equilibrium, Market completeness, Allocational equivalence

JEL classification: D51, D61, D91 


\section{Introduction}

Debreu (1959) discusses how the one-period Arrow-Debreu (1954) model can be used to analyze multi-period settings with uncertainty. The crucial idea is to include the period and the state of the world at the time of delivery in the definition of a commodity, resulting in contingent commodities. Debreu assumes that markets are complete in the sense that all contingent commodities can be traded in the first period and does not allow for markets to reopen after the first period. It is indeed widely believed that there is no need for markets to reopen as time passes.

As an alternative to the assumption that all contingent commodities can be traded in the initial period, sequentially complete markets have been considered. In a sequentially complete market structure, it is typically assumed that at every state there are complete spot markets for commodities available at that state together with a restricted set of markets for commodities available at future states. It is well-known that, under certain assumptions, equilibria in the Arrow-Debreu model are allocationally equivalent to equilibria in sequentially complete models, see Arrow(1953). Donaldson and Selden (1981) and Haller (1990) provide two discussions of the assumptions needed for this result. Drèze and Herings (2003) show in an example that there might be equilibrium continuations in a sequentially complete markets setting that are not allocationally equivalent to any equilibrium in the Arrow-Debreu setting. A closer look at their example suggests that the result is driven by the fact that in the Arrow-Debreu setting, markets are not reopened. Chattopadhyay and Gottardi (1999) consider differences between complete and sequentially complete market structures in overlapping generations models.

This paper studies multi-period economies subject to uncertainty, where markets may reopen at future states. At every state, households are endowed with preferences that may or may not be time-consistent. In general equilibrium models, time-inconsistent preferences have been introduced by Luttmer and Mariotti (2003) and Herings and Rohde (2004). We consider three market structures that are commonly believed to result in the same set of competitive equilibria. First, we consider the Arrow-Debreu structure with complete markets in the first period and no reopening of markets as time passes. Second, we consider sequentially complete markets, where in every state, people can trade the goods to be consumed in that state and all one-period ahead contingent commodities. Finally, we consider the complete market structure. We say that markets are complete if, in every state, there are complete markets for all contingent commodities to be consumed in that state or in a later state.

We follow Pollak (1968) in making a distinction between naïve and sophisticated behavior. Sophisticated households make plans that they expect to stick to in the future. These households can be interpreted as consisting of multiple agents, playing a subgame perfect equilibrium against themselves.

In general, the Arrow-Debreu market structure leads to equilibrium allocations that differ from equilibrium allocations corresponding to the other two market structures. The explanation is that the Arrow-Debreu market structure yields the commitment power needed not to re-trade. For sophisticated economies, sequentially complete markets yield the 
same allocations as complete markets do, even when preferences are allowed to be timeinconsistent. The assumption of sophisticated behavior is extremely demanding. It might be more reasonable to assume that households, at least partly, mispredict their future preferences (Loewenstein, O'Donoghue, and Rabin (2003)). For naïve economies any sequentially complete equilibrium is allocationally equivalent to some complete equilibrium, but the converse statement does not hold.

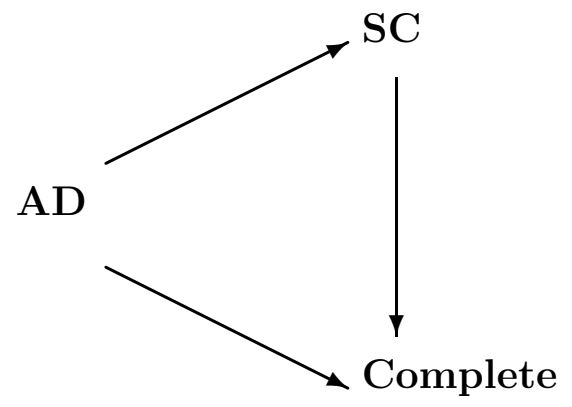

Figure 1: Naïve equilibria when preferences are time-consistent.

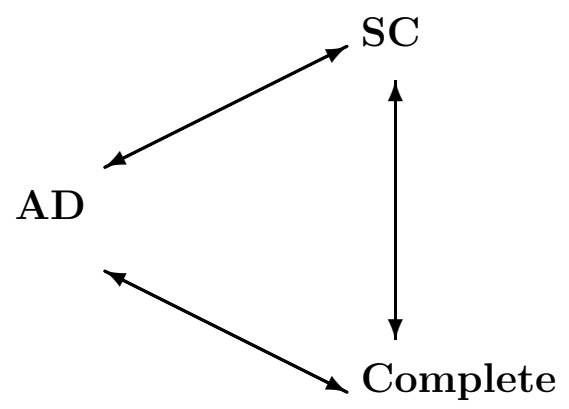

Figure 2: Sophisticated equilibria when preferences are time-consistent.

When preferences are time-consistent, we show that all three markets structures are allocationally equivalent for sophisticated economies. For naïve economies, every equilibrium in the Arrow-Debreu market structure is allocationally equivalent to some sequentially complete equilibrium and some complete equilibrium, but surprisingly the reverse does not hold. The results of this paper are summarized in Figures 1, 2, 3 and 4. In those figures an arrow from ' $\mathrm{A}$ ' to ' $\mathrm{B}$ ' indicates that any concept ' $\mathrm{A}$ ' equilibrium allocation is allocationally equivalent to some concept ' $\mathrm{B}$ ' equilibrium allocation.

This paper therefore identifies a crucial assumption that has to be satisfied in order for complete and sequentially complete markets to be equivalent: behavior should be sophisticated. For equivalence to Arrow-Debreu markets, it is also needed that preferences are time-consistent.

Section 2 starts by describing the primitives of the economies as considered in this paper, without elaborating on market structures. Section 3 describes the Arrow-Debreu 
$\mathrm{AD}$

Complete

Figure 3: Naïve equilibria when preferences are time-inconsistent.

$\mathrm{AD}$

$\mathrm{SC}$

Complete

Figure 4: Sophisticated equilibria when preferences are time-inconsistent.

market structure. Sections 4 and 5 analyze the sequentially complete markets structure and the complete one, respectively. Both these sections are built up in the same fashion. First, naïve and sophisticated behavior is described. Then, properties of the specific market structure are studied. Section 6 then concludes.

\section{The Primitives of the Economy}

There is a finite number of periods $t \leq T$, where $t, T \in \mathbb{N}$. In every period $t \geq 2,|\Omega|$ possible events can happen, where $\Omega$ denotes a finite set of events. A sequence of $T$ events constitutes a state. We denote $S=\Omega^{T-1}$. For every $s \in S$ and every $t \in T$ with $t \geq 2$, we define the state at period $t$ by

$$
s(t)=\left\{\left(\omega_{\tau}\right)_{\tau=2}^{t} \in \Omega^{t-1} \mid \exists\left(\omega_{\tau}\right)_{\tau=t+1}^{T} \in \Omega^{T-t} \text { with }\left(\omega_{\tau}\right)_{\tau=2}^{T}=s\right\} .
$$

For $t=1$, we define $s(1)=1$.

Figure 5 illustrates the structure of an economy with 4 periods $(T=4)$ and two events in every period $(\Omega=\{\operatorname{Bad}, \operatorname{Good}\})$. A state in period 4 is a path from the root of the tree to an end-node. Thus, in the figure there are 8 states in period 4 . Consider the state that corresponds to the path that leads to the third end-node from above. We call this state 


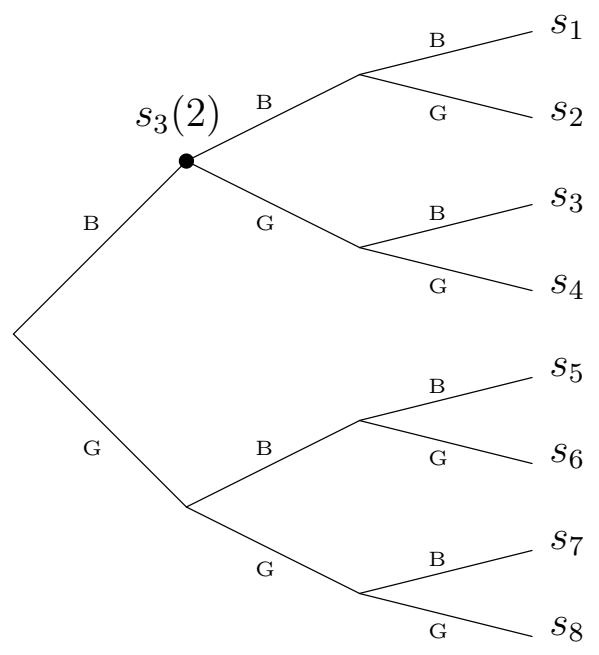

Figure 5: An economy with $T=4$ and $\Omega=\{\operatorname{Bad}$, Good $\}$

$s_{3}=(B, G, B)$. We have $s_{3}(2)=B, s_{3}(3)=(B, G)$, and $s_{3}(4)=(B, G, B)$. For every $s(t)$ we define $s^{+}(t)=\left\{\sigma(t+1) \in \Omega^{t} \mid \sigma \in S, \sigma(t)=s(t)\right\}$.

The economy consists of a finite number of households $h \in H$. In every state of every period, households consume a finite number of goods $l \in L$. For $s \in S$ and $\tau \geq t$, the consumption bundle $x_{s(\tau) \mid s(t)}^{h}$ denotes the consumption of every good at state $s(\tau)$ anticipated in state $s(t)$. Consumption for state $s(t)$ and every later state anticipated at $s(t)$, is given by the consumption plan $x_{\cdot \mid s(t)}^{h}$. The notation $x_{\cdot \mid s(t)}^{h}\left\langle\hat{x}_{\cdot \mid s(\tau)}^{h}\right.$, means $x_{\cdot \mid s(t)}^{h}$ with $x_{s(\tau), T \mid s(t)}^{h}$ replaced by $\hat{x}_{\cdot \mid s(\tau)}^{h}$. For $t \in T$, we define $\mathcal{L}_{t}=L \sum_{\tau=1}^{T-t}|\Omega|^{\tau}$, the total number of commodities to be consumed at states later than $s(t)$. Consumption in every state is limited to the consumption sets $X_{\cdot \mid s(t)}^{h} \subset \mathbb{R}^{L+\mathcal{L}_{t}}$, where the interpretation of sub- and superscripts is similar as before. A subscript $l$ is added to denote consumption of good $l$. In every state $s(t)$, households have initial endowments $e_{\cdot \mid s(t)}^{h}$. Households are assumed to have correct expectations about consumption sets and endowments.

In every state, households have preferences over consumption in that state and all possible future states. Preferences of household $h$ in state $s(t)$ are represented by $\succeq^{h, s(t)}$, a preference relation defined on $X_{\cdot \mid s(t)}^{h}$. The complete set of preferences of household $h$ is denoted by $\succeq^{h}=\left(\succeq^{h, s(t)}\right)_{s \in S, t \in T}$.

Preferences might depend on the state of nature and can change over time. Consider a consumption plan in state $s(t)$. If we replace consumption from a future state $\sigma(\tau)$ on by consumption that will be preferred once state $\sigma(\tau)$ is reached, then time-consistency of preferences implies that the resulting consumption plan at state $s(t)$ is preferred to the original one.

\section{Definition 2.1 Time-consistent Preferences}


Preferences for household $h$ are time-consistent if for all periods $t, \tau$ with $\tau \geq t$ and all states $s, \sigma \in S$ with $\sigma(t)=s(t)$, for every $x_{\cdot \mid s(t)}^{h} \in X_{\cdot \mid s(t)}^{h}$ and every $\widehat{x}_{\cdot \mid \sigma(\tau)}^{h} \in X_{\cdot \mid \sigma(\tau)}^{h}$, we have

$$
x_{\cdot \mid s(t)}^{h} \succeq^{h, s(t)} \quad x_{\cdot \mid s(t)}^{h}\left\langle\widehat{x}_{\cdot \mid \sigma(\tau)}^{h}\right.
$$

if and only if

$$
x_{\sigma(\tau), T \mid s(t)}^{h} \succeq^{h, \sigma(\tau)} \quad \widehat{x}_{\cdot \mid \sigma(\tau)}^{h} .
$$

Preferences are time-inconsistent if they are not time-consistent.

Preferences of a household in a particular state $s(t)$ are locally non-satiated in state $s\left(t^{\prime}\right)$, where $t^{\prime} \geq t$, if for every consumption plan in state $s(t)$, we can find another consumption plan in state $s(t)$ that (i) only deviates from the initial consumption plan in state $s\left(t^{\prime}\right)$, (ii) is sufficiently close to the initial consumption plan, and (iii) is preferred to the initial consumption plan. This can be formalized as follows. Consider a household $h$, a state $s$, and two periods $t, t^{\prime}$ with $t^{\prime} \geq t$. Preferences $\succeq^{h, s(t)}$ are locally non-satiated in $s\left(t^{\prime}\right)$ if for every $x_{\cdot \mid s(t)}^{h} \in X_{\cdot \mid s(t)}^{h}$, for every $\varepsilon>0$, there is an $\bar{x}_{\cdot \mid s(t)}^{h} \in X_{\cdot \mid s(t)}^{h}$ with $\bar{x}_{\sigma(\tau) \mid s(t)}^{h}=x_{\sigma(\tau) \mid s(t)}^{h}$ for every $\sigma(\tau) \neq s\left(t^{\prime}\right),\left\|\bar{x}_{\cdot \mid s(t)}^{h}-x_{\cdot \mid s(t)}^{h}\right\|<\varepsilon$ and $\bar{x}_{\cdot{ }_{\mid s(t)}^{h}} \succ^{h, s(t)} x_{\cdot \mid s(t)}^{h}$. An economy with preferences $\left(\succeq^{h, s(t)}\right)_{h \in H, s \in S, t \in T}$ is locally non-satiated if for every state $s$ and every $t, t^{\prime}$ with $t^{\prime} \geq t$ there is a household $h$ such that $\succeq^{h, s(t)}$ is locally non-satiated in $s\left(t^{\prime}\right)$.

An economy $\mathcal{E}$ is described by its primitives $\left(X_{. \mid 1}^{h}, e_{. \mid 1}^{h}, \succeq^{h}\right)_{h \in H}$. A household will sell its endowments and use the revenues from this sale to buy goods he desires most. The timing of the opportunities to sell endowments will depend on the market structure.

\section{Arrow-Debreu Markets}

This section considers the model of Arrow and Debreu (1954) and Debreu (1959). There are complete markets in contingent commodities in period 1. Markets do not reopen in later periods. All commodities can be traded in period 1 against the prices $p_{\cdot \mid 1}$. Prices for commodities in state $s(t)$ are then given by $p_{s(t) \mid 1}$. Prices for state $s(t)$ and all states that can be reached from $s(t)$ are denoted by $p_{s(t), T \mid 1}$. We denote the set of possible prices by $P_{. \mid 1}=\mathbb{R}^{L+\mathcal{L}_{1}}$.

In the Arrow-Debreu model, markets do not reopen after period 1. Therefore, it is implicitly assumed that consumption in those later periods is completely determined by consumption in period 1. Moreover, since markets do not reopen after period 1 , there is no need to specify prices in periods after period 1. To be consistent in notation throughout this paper, we do want to specify prices in periods after period 1. Without loss of generality, we set prices in states later than period 1 equal to the prices in period 1 of corresponding contingent commodities. We define $p_{\cdot \mid s(t)}=p_{s(t), T \mid 1}$ for every $s(t)$, and $P_{\cdot \mid s(t)}=\mathbb{R}^{L+\mathcal{L}_{t}}$ and $P=\prod_{s(t)} P_{\cdot \mid s(t)}$.

The opportunity set of household $h$ in period 1 is given by

$$
\breve{\gamma}_{1}^{h}\left(p_{\cdot \mid 1}, e_{\cdot \mid 1}^{h}\right)=\left\{x_{\cdot \mid 1}^{h} \in X_{\cdot \mid 1}^{h} \mid p_{\cdot \mid 1} x_{\cdot \mid 1}^{h} \leq p_{\cdot \mid 1} e_{\cdot \mid 1}^{h}\right\} .
$$


The opportunity set of household $h$ in any state $s(t)$ with $t>1$ is given by

$$
\breve{\gamma}_{s(t)}^{h}\left(p_{\cdot \mid s(t)}, x_{s(t), T \mid s(t-1)}^{h}\right)=\left\{x_{s(t), T \mid s(t-1)}^{h}\right\} .
$$

A household selects a most preferred consumption plan in the opportunity set. Thus, the demand sets are given by

$$
\begin{aligned}
\breve{\delta}_{s(t)}^{h}\left(p_{\cdot \mid s(t)}, x_{s(t), T \mid s(t-1)}^{h}\right)= & \left\{\bar{x}_{\cdot \mid s(t)}^{h} \in \breve{\gamma}_{s(t)}^{h}\left(p_{\cdot \mid s(t)}, x_{s(t), T \mid s(t-1)}^{h}\right) \mid\right. \\
& \left.\quad \nexists x_{\cdot \mid s(t)}^{h} \in \breve{\gamma}_{s(t)}^{h}\left(p_{\cdot \mid s(t)}, x_{s(t), T \mid s(t-1)}^{h}\right) \text { with } x_{\cdot \mid s(t)}^{h} \succ^{h, s(t)} \bar{x}_{\cdot \mid s(t)}^{h}\right\} .
\end{aligned}
$$

In equilibrium, total demand must equal total endowments. For notational purposes, we define $x_{1, T \mid s(0)}^{* h}=e_{\cdot \mid 1}^{h}$.

\section{Definition 3.1 Arrow-Debreu (AD) Equilibrium}

A pair $\left(p^{*}, x^{*}\right) \in P \times X$ is an Arrow-Debreu (AD) equilibrium of the economy $\mathcal{E}$ if

(a) $x_{\cdot \mid s(t)}^{* h} \in \breve{\delta}_{s(t)}^{h}\left(p_{\cdot \mid s(t)}^{*}, x_{s(t), T \mid s(t-1)}^{* h}\right)$ for all $h \in H, t \in T, s \in S$,

(b) $\sum_{h \in H} x_{\cdot \mid s(t)}^{* h}=\sum_{h \in H} e_{\cdot \mid s(t)}^{h}$ for all $t \in T, s \in S$,

(c) $p_{\cdot \mid s(t)}^{*}=p_{s(t), T \mid 1}^{*}$ for all $t \in T, s \in S$.

\section{Sequentially Complete Markets}

This section considers sequentially complete markets. In every state there are spot markets and forward markets for one-period ahead contingent commodities. In period $t$ households can trade all goods for period $t$ and all goods for all possible events in period $t+1$. They have to form expectations about prices for goods that will be consumed in later periods, i.e. periods from $t+2$ on. We assume that they form point expectations and that all households form the same expectations. We let $P_{\cdot \mid s(t)}=\mathbb{R}^{L+\mathcal{L}_{t}}, \bar{Q}_{\cdot \mid s(t)}=\mathbb{R}^{\mathcal{L} t}$, $P=\prod_{s(t)} P_{\cdot \mid s(t)}$ and $\bar{Q}=\prod_{s(t)} \bar{Q}_{\cdot \mid s(t)}$ Prices and expected (at state $s(t)$ ) prices on spot markets will be denoted by $p_{\cdot \mid s(t)} \in P_{\cdot \mid s(t)}$. Prices and expected (at state $s(t)$ ) prices of forward commodities will be denoted by $q_{\cdot \mid s(t)} \in \bar{Q}_{\cdot \mid s(t)}$. The (expected) prices in state $s(t)$ of commodities to be delivered in state $s(\tau)$ and traded in $s(\tau-1)$, are denoted by $q_{s(\tau) \mid s(t)}$. We define $\bar{Y}_{\cdot \mid s(t)}^{h}=\mathbb{R}^{\mathcal{L}_{t}}$ and $\bar{Y}=\prod_{h \in H} \prod_{s(t)} \bar{Y}_{\cdot \mid s(t)}^{h}$. For household $h$ the forward commodity bundle for state $s(\tau)$ that is expected (in state $s(t)$ ) to be bought in state $s(\tau-1)$ is denoted by $y_{s(\tau) \mid s(t)}^{h}$. For notational purposes, we define $y_{\cdot \mid s(0)}^{h}=e_{\cdot \mid 1}^{h}$.

When markets can reopen in the future, and preferences are allowed to be timeinconsistent, a conflict can arise between the preferences from the perspective of one state and those of another, later, state. Following Pollak (1968), we make a distinction between naïve and sophisticated households. Naïve households will typically revise their plans over time. Sophisticated households only make choices that they expect to stick to in the future. The latter have the additional constraint that their expected future consumption should be in their future demand sets given expected future prices. First, we consider naïve and then sophisticated behavior. 


\subsection{Naïve Behavior}

In every state, naïve households sell the endowments of that particular state and the oneperiod ahead endowments. They use the revenue from selling these commodities to buy goods on the spot markets and to buy one-period ahead contingent commodities. At each state $s(t)$ households form expectations about all future prices.

The opportunity set of household $h$ in state $s(t)$ is given by

$$
\begin{gathered}
n \bar{\gamma}_{s(t)}^{h}\left(p_{\cdot \mid s(t)}, q_{\cdot \mid s(t)}, y_{s(t) \mid s(t-1)}^{h}, e_{t+1, T \mid s(t)}^{h}\right)=\left\{\left(x_{\cdot \mid s(t)}^{h}, y_{\cdot \mid s(t)}^{h}\right) \in X_{\cdot \mid s(t)}^{h} \times \bar{Y}_{\cdot \mid s(t)}^{h} \mid\right. \\
p_{\sigma(\tau) \mid s(t)} x_{\sigma(\tau) \mid s(t)}^{h}+\sum_{\sigma(\tau+1) \in \sigma^{+}(\tau)} q_{\sigma(\tau+1) \mid s(t)} y_{\sigma(\tau+1) \mid s(t)}^{h} \leq \\
p_{\sigma(\tau) \mid s(t)} y_{\sigma(\tau) \mid s(t)}^{h}+\sum_{\sigma(\tau+1) \in \sigma^{+}(\tau)} q_{\sigma(\tau+1) \mid s(t)} e_{\sigma(\tau+1) \mid s(t)}^{h} \\
\text { for every } \tau \geq t \text {, and every } \sigma(\tau) \text { with } \sigma(t)=s(t)\},
\end{gathered}
$$

where, by definition, $y_{s(t) \mid s(t)}^{h}=y_{s(t) \mid s(t-1)}^{h}$ and $y_{1 \mid 0}^{h}=e_{1 \mid 0}^{h}$.

The demand set of household $h$ in state $s(t)$ is then given by

$$
\begin{gathered}
{ }^{n} \bar{\delta}_{s(t)}^{h}\left(p_{\cdot \mid s(t)}, q_{\cdot \mid s(t)}, y_{s(t) \mid s(t-1)}^{h}, e_{t+1, T \mid s(t)}^{h}\right)=\left\{\left(\bar{x}_{\cdot \mid s(t)}^{h}, \bar{y}_{\cdot \mid s(t)}^{h}\right) \in{ }^{n} \bar{\gamma}_{s(t)}^{h}\left(p_{\cdot \mid s(t)}, q_{\cdot \mid s(t)}, y_{s(t) \mid s(t-1)}^{h}, e_{t+1, T \mid s(t)}^{h}\right) \mid\right. \\
\left.\nexists\left(x_{\cdot \mid s(t)}^{h}, y_{\cdot \mid s(t)}^{h}\right) \in{ }^{n} \bar{\gamma}_{s(t)}^{h}\left(p_{\cdot \mid s(t)}, q_{\cdot \mid s(t)}, y_{s(t) \mid s(t-1)}^{h}, e_{t+1, T \mid s(t)}^{h}\right) \text { with } x_{\cdot \mid s(t)}^{h} \succ^{h, s(t)} \bar{x}_{\cdot \mid s(t)}^{h}\right\} .
\end{gathered}
$$

Prices and consumption are said to constitute a naïve sequentially complete equilibrium if consumption is in the demand sets and if markets clear and are expected to clear in every state.

\section{Definition 4.1 Naïve Sequentially Complete (SC) Equilibrium}

A pair $\left(p^{*}, q^{*}, x^{*}, y^{*}\right) \in P \times \bar{Q} \times X \times \bar{Y}$ is a Naïve Sequentially Complete (SC) equilibrium of the economy $\mathcal{E}$ if

(a) $\left(x_{\cdot \mid s(t)}^{* h}, y_{\cdot \mid s(t)}^{* h}\right) \in{ }^{n} \bar{\delta}_{s(t)}^{h}\left(p_{\cdot \mid s(t)}^{*}, q_{\cdot \mid s(t)}^{*}, y_{s(t) \mid s(t-1)}^{* h}, e_{t+1, T \mid s(t)}^{h}\right)$ for all $h \in H, t \in T, s \in S$,

(b) $\sum_{h \in H} x_{\cdot \mid s(t)}^{* h}=\sum_{h \in H} e_{\cdot \mid s(t)}^{h}$ for all $t \in T, s \in S$, and

(c) $\sum_{h \in H} y_{\cdot \mid s(t)}^{* h}=\sum_{h \in H} e_{\cdot \mid s(t+1)}^{h}$ for all $t \in T, s \in S$.

\subsection{Sophisticated Behavior}

Sophisticated households only make plans that they expect to adhere to in the future. They face the additional constraint that their expected future consumption should be in their future demand sets given expected future prices. 
The opportunity set of sophisticated household $h$ in state $s(T)$ is the same as for its naïve counterpart:

$$
{ }^{s} \bar{\gamma}_{s(T)}^{h}\left(p_{\cdot \mid s(T)}, y_{s(T) \mid s(T-1)}^{h}\right)={ }^{n} \bar{\gamma}_{s(T)}^{h}\left(p_{\cdot \mid s(T)}, y_{s(T) \mid s(T-1)}^{h}\right) .
$$

The opportunity set of sophisticated household $h$ in state $s(t)$, where $t<T$, is given by

$$
s \bar{\gamma}_{s(t)}^{h}\left(p_{\cdot \mid s(t)}, q \cdot \mid s(t), y_{s(t) \mid s(t-1)}^{h}, e_{t+1, T \mid s(t)}^{h}\right)=\left\{\left(x_{\cdot \mid s(t)}^{h}, y_{\cdot \mid s(t)}^{h}\right) \in X_{\cdot \mid s(t)}^{h} \times \bar{Y}_{\cdot \mid s(t)}^{h} \mid\right.
$$

(i)

$$
\begin{aligned}
& p_{\sigma(\tau) \mid s(t)} x_{\sigma(\tau) \mid s(t)}^{h}+\sum_{\sigma(\tau+1) \in \sigma^{+}(\tau)} q_{\sigma(\tau+1) \mid s(t)} y_{\sigma(\tau+1) \mid s(t)}^{h} \leq \\
& p_{\sigma(\tau) \mid s(t)} y_{\sigma(\tau) \mid s(t)}^{h}+\sum_{\sigma(\tau+1) \in \sigma^{+}(\tau)} q_{\sigma(\tau+1) \mid s(t)} e_{\sigma(\tau+1) \mid s(t)}^{h}
\end{aligned}
$$

for every $\tau \geq t$, and every $\sigma(\tau)$ with $\sigma(t)=s(t)$,

and

(ii) $\left(x_{s(t+1), T \mid s(t)}^{h}, y_{s^{+}(t+1), T \mid s(t)}^{h}\right) \in$

$$
s \bar{\delta}_{s(t+1)}^{h}\left(p_{s(t+1), T \mid s(t)}, q_{s^{+}(t+1), T \mid s(t)}, y_{s(t+1) \mid s(t)}^{h}, e_{t+2, T \mid s(t+1)}^{h}\right)
$$

for every $\left.s(t+1) \in s^{+}(t)\right\}$.

The demand set of a sophisticated household is given by

$$
\begin{gathered}
s \bar{\delta}_{s(t)}^{h}\left(p_{\cdot \mid s(t)}, q_{\cdot \mid s(t)}, y_{s(t) \mid s(t-1)}^{h}, e_{t+1, T \mid s(t)}^{h}\right)=\left\{\left(\bar{x}_{\cdot \mid s(t)}^{h}, \bar{y}_{\cdot \mid s(t)}^{h}\right) \in{ }^{s} \bar{\gamma}_{s(t)}^{h}\left(p_{\cdot \mid s(t)}, q_{\cdot \mid s(t)}, y_{s(t) \mid s(t-1)}^{h}, e_{t+1, T \mid s(t)}^{h}\right) \mid\right. \\
\left.\nexists\left(x_{\cdot \mid s(t)}^{h}, y_{\cdot \mid s(t)}^{h}\right) \in{ }^{s} \bar{\gamma}_{s(t)}^{h}\left(p_{\cdot \mid s(t)}, q_{\cdot \mid s(t)}, y_{s(t) \mid s(t-1)}^{h}, e_{t+1, T \mid s(t)}^{h}\right) \text { with } x_{\cdot \mid s(t)}^{h} \succ^{h, s(t)} \bar{x}_{\cdot \mid s(t)}^{h}\right\} .
\end{gathered}
$$

Prices, consumption bundles and purchases of forward commodities constitute a sophisticated sequentially complete equilibrium if actual and planned supply of spot and forward commodities equals demand, price expectations are correct, and expected consumption and expected purchases of forward commodities equal realized consumption and realized purchases of forward commodities. This is formalized in the following definition.

\section{Definition 4.2 Sophisticated Sequentially Complete (SC) Equilibrium}

A pair $\left(p^{*}, q^{*}, x^{*}, y^{*}\right) \in P \times \bar{Q} \times X \times \bar{Y}$ is a Sophisticated Sequentially Complete (SC) equilibrium of the economy $\mathcal{E}$ if

(a) $\left(x_{\cdot \mid s(t)}^{* h}, y_{\cdot \mid s(t)}^{* h}\right) \in{ }^{s} \bar{\delta}_{s(t)}^{h}\left(p_{\cdot \mid s(t)}^{*}, q_{\cdot \mid s(t)}^{*}, y_{s(t) \mid s(t-1)}^{* h}, e_{t+1, T \mid s(t)}^{h}\right)$ for all $h \in H, t \in T, s \in S$,

(b) $\sum_{h \in H} x_{\cdot \mid s(t)}^{* h}=\sum_{h \in H} e_{\cdot \mid s(t)}^{h}$ for all $t \in T, s \in S$,

(c) $\sum_{h \in H} y_{\cdot \mid s(t)}^{* h}=\sum_{h \in H} e_{\cdot \mid s(t+1)}^{h}$ for all $t \in T, s \in S$,

(d) $p_{s(\tau), T \mid s(t)}^{*}=p_{\cdot \mid s(\tau)}^{*}$ for all $s \in S, t, \tau \in T$ with $t \leq \tau$,

(e) $q_{s(\tau), T \mid s(t)}^{*}=q_{s(\tau), T \mid s(\tau-1)}^{*}$ for all $s \in S, t, \tau \in T$ with $t<\tau$,

(f) $x_{s(\tau), T \mid s(t)}^{* h}=x_{\cdot \mid s(\tau)}^{* h}$ for all $h \in H, s \in S, t, \tau \in T$ with $t \leq \tau$, and

(g) $y_{s(\tau), T \mid s(t)}^{* h}=y_{s(\tau), T \mid s(\tau-1)}^{* h}$ for all $h \in H, s \in S, t, \tau \in T$ with $t<\tau$. 


\subsection{Properties of Sequentially Complete Equilibria}

In both naïve SC and sophisticated SC equilibria, expectations of relative prices on spot markets are equal to the corresponding expected relative prices of assets or one-period ahead contingent commodities, except when all those asset prices are equal to zero. This is formalized in the following lemma. Svensson (1976) derives a similar result in a two-period economy without uncertainty. He called these expectations stationary point expectations.

Theorem 4.3 Let the economy $\mathcal{E}$ be locally non-satiated and let $\left(p^{*}, q^{*}, x^{*}, y^{*}\right)$ be a naïve $S C$ equilibrium of $\mathcal{E}$. Then for every $s, \sigma \in S$ and every $t, \tau \in T$ with $\tau>t$ and $\sigma(t)=s(t)$ there is $\mu_{\sigma(\tau) \mid s(t)} \in \mathbb{R}$ such that

$$
q_{\sigma(\tau) \mid s(t)}^{*}=\mu_{\sigma(\tau) \mid s(t)} p_{\sigma(\tau) \mid s(t)}^{*}
$$

\section{Proof}

Let $\left(p^{*}, q^{*}, x^{*}, y^{*}\right)$ be a naïve SC equilibrium. Consider some $s, \sigma \in S$ and $\tau, t \in T$ with $\tau>$ $t$ and $\sigma(t)=s(t)$. Since the economy is locally non-satiated, it is evident that $p_{\sigma(\tau) \mid s(t)}^{*} \neq 0$. Let $l$ be a commodity such that $p_{\sigma(\tau), l \mid s(t)}^{*} \neq 0$.

Suppose there is $\tilde{l} \in L$ such that there is no $\mu \in \mathbb{R}$ with both

$$
\begin{aligned}
& \mu p_{\sigma(\tau), l \mid s(t)}^{*}=q_{\sigma(\tau), l \mid s(t)}^{*} \text { and } \\
& \mu p_{\sigma(\tau), \tilde{l} \mid s(t)}^{*}=q_{\sigma(\tau), \tilde{l} \mid s(t)}^{*} .
\end{aligned}
$$

Then it follows that

$$
p_{\sigma(\tau), l \mid s(t)}^{*} q_{\sigma(\tau), \tilde{l} \mid s(t)}^{*} \neq p_{\sigma(\tau), \tilde{l} \mid s(t)}^{*} q_{\sigma(\tau), l \mid s(t)}^{*}
$$

Let $h$ be a household such that $\succeq^{h, s(t)}$ is locally non-satiated in $\sigma(\tau-1)$.

Case 1: Assume that

$$
p_{\sigma(\tau), l \mid s(t)}^{*} q_{\sigma(\tau), \tilde{l} \mid s(t)}^{*}>p_{\sigma(\tau), \tilde{l} \mid s(t)}^{*} q_{\sigma(\tau), l \mid s(t)}^{*}
$$

Now consider the vector $\Delta y_{\cdot \mid s(t)}^{h}$ defined by

$$
\Delta y_{s^{\prime}\left(t^{\prime}\right), l^{\prime} \mid s(t)}^{h}= \begin{cases}p_{\sigma(\tau), \tilde{l} \mid s(t)}^{*} & \text { if } s^{\prime}\left(t^{\prime}\right)=\sigma(\tau) \text { and } l^{\prime}=l \\ -p_{\sigma(\tau), l \mid s(t)}^{*} & \text { if } s^{\prime}\left(t^{\prime}\right)=\sigma(\tau) \text { and } l^{\prime}=\tilde{l} \\ 0 & \text { otherwise }\end{cases}
$$

Then

$$
p_{\sigma(\tau) \mid s(t)}^{*} \Delta y_{\sigma(\tau) \mid s(t)}^{h}=0
$$

i.e. in state $s(t)$ household $h$ could plan to buy $\Delta y_{\cdot \mid s(t)}^{h}$ in addition to $y_{\cdot \mid s(t)}^{* h}$, without changing income in state $\sigma(\tau)$. Moreover,

$$
q_{\sigma(\tau) \mid s(t)}^{*} \Delta y_{\sigma(\tau) \mid s(t)}^{h}=q_{\sigma(\tau), l \mid s(t)}^{*} p_{\sigma(\tau), \tilde{l} \mid s(t)}^{*}-q_{\sigma(\tau), \tilde{l} \mid s(t)}^{*} p_{\sigma(\tau), l \mid s(t)}^{*}<0 .
$$


Therefore, buying $\Delta y_{\cdot \mid s(t)}^{h}$ in addition to $y_{\cdot \mid s(t)}^{* h}$ decreases expected expenditures in state $\sigma(\tau-1)$, while not changing income in any other state.

By local non-satiation this contradicts $\left(p^{*}, q^{*}, x^{*}, y^{*}\right)$ being a naïve $\mathrm{SC}$ equilibrium. Case 2: Assume that

$$
p_{\sigma(\tau), l \mid s(t)}^{*} q_{\sigma(\tau), \tilde{l} \mid s(t)}^{*}<p_{\sigma(\tau), \tilde{l} \mid s(t)}^{*} q_{\sigma(\tau), l \mid s(t)}^{*}
$$

This case follows by repeating the argument of Case 1, replacing $\Delta y_{\cdot \mid s(t)}^{h}$ by $-\Delta y_{\left.\cdot\right|_{s(t)} ^{h}}^{h}$

It follows that for any $\tilde{l} \in L, \mu=q_{\sigma(\tau), l \mid s(t)}^{*} / p_{\sigma(\tau), l \mid s(t)}^{*}$ satisfies $\mu p_{\sigma(\tau), \tilde{l} \mid s(t)}^{*}=q_{\sigma(\tau), \tilde{l} \mid s(t)}^{*}$. Q.E.D.

The result of Theorem 4.3 is driven by the arbitrage opportunities that would be created in locally non-satiated economies if the condition of the result would be violated. This intuition is entirely correct for the naïve case. The sophisticated case is more difficult. The reason is that a sophisticated household cannot guarantee that his future self will behave as he would like him to behave. Therefore, changing income in a future state may make the future self consume a bundle that the current self does not like at all. The availability of an arbitrage opportunity in a particular state is not sufficient to drive the result. The arbitrage opportunity needs to be such that also income in future states is unchanged, so that future selves will behave the same, irrespective of whether the arbitrage opportunity is taken or not. Our proof for the naïve case is constructed in such a way that these properties are satisfied, and applies therefore to the sophisticated case as well.

Theorem 4.4 Let the economy $\mathcal{E}$ be locally non-satiated and let $\left(p^{*}, q^{*}, x^{*}, y^{*}\right)$ be a sophisticated $S C$ equilibrium of $\mathcal{E}$. Then for every $s, \sigma \in S$ and every $t, \tau \in T$ with $\tau>t$ and $\sigma(t)=s(t)$ there is a $\mu_{\sigma(\tau) \mid s(t)} \in \mathbb{R}$ such that

$$
q_{\sigma(\tau) \mid s(t)}^{*}=\mu_{\sigma(\tau) \mid s(t)} p_{\sigma(\tau) \mid s(t)}^{*}
$$

An $\mathrm{AD}$ equilibrium $\left(p^{*}, q^{*}\right)$ is said to be allocationally equivalent to a naïve (sophisticated) SC equilibrium if there are $p \in P, q \in \bar{Q}$ and $y \in \bar{Y}$ such that $\left(p, q, x^{*}, y\right)$ is a naïve (sophisticated) SC equilibrium. Similarly, a naïve (sophisticated) SC equilibrium $\left(p^{*}, q^{*}, x^{*}, y^{*}\right)$ is said to be allocationally equivalent to an $\mathrm{AD}$ equilibrium if there is $p \in P$ such that $\left(p, x^{*}\right)$ is an $\mathrm{AD}$ equilibrium.

When preferences are time-consistent one might expect that there is no difference between naïve and sophisticated behavior. However, this is not the case. Naïve SC equilibria are not necessarily allocationally equivalent to AD equilibria. However, sophisticated SC equilibria are allocationally equivalent to AD equilibria and the other way around. Moreover, every $\mathrm{AD}$ equilibrium is allocationally equivalent to some $\mathrm{SC}$ equilibrium in both the naïve and the sophisticated case.

The following example, that shows that even for time-consistent preferences, there are naïve SC equilibria that are not allocationally equivalent to any $\mathrm{AD}$ equilibrium, is borrowed from Drèze and Herings (2003). 
Example 4.5 Consider a two-period economy without uncertainty. There are two agents and two goods in every period. Endowments are $e_{\cdot \mid 1}^{h}=(2,2,2,2)$ for $h=1,2$. Preferences are time-consistent and represented by

$$
u_{1}^{h}\left(x_{\cdot \mid 1}^{h}\right)=v^{h}\left(x_{1, \mid 11}^{h}, x_{1,2 \mid 1}^{h}\right)^{\frac{1}{2}} \cdot v^{h}\left(x_{2,1 \mid 1}^{h}, x_{2,2 \mid 1}^{h}\right)^{\frac{1}{2}},
$$

where

$$
v^{1}\left(x_{\cdot, 1 \mid 1}^{1}, x_{\cdot, 2 \mid 1}^{1}\right)= \begin{cases}{\left[\min \left(x_{\cdot, 1 \mid 1}^{1}, x_{\cdot, 2 \mid 1}^{1}\right)\right],} & \min \left(x_{\cdot, 1 \mid 1}^{1}, x_{\cdot, 2 \mid 1}^{1}\right) \leq 1, \\ {\left[\left(x_{\cdot, 1 \mid 1}^{1}-1\right)^{\frac{1}{2}}\left(x_{\cdot, 2 \mid 1}^{1}-1\right)^{\frac{1}{2}}+1\right],} & \min \left(x_{\cdot, 1 \mid 1}^{1}, x_{\cdot, 2 \mid 1}^{1}\right) \geq 1,\end{cases}
$$

and

$$
v^{2}\left(x_{\cdot, 1 \mid 1}^{2}, x_{\cdot, 2 \mid 1}^{2}\right)= \begin{cases}{\left[\min \left(x_{\cdot, 1 \mid 1}^{2}, x_{\cdot, 2 \mid 1}^{2}\right)\right],} & \min \left(x_{\cdot, 1 \mid 1}^{2}, x_{\cdot, 2 \mid 1}^{2}\right) \leq 3, \\ {\left[\left(x_{\cdot, 1 \mid 1}^{2}-3\right)^{\frac{1}{2}}\left(x_{\cdot, 2 \mid 1}^{2}-3\right)^{\frac{1}{2}}+3\right],} & \min \left(x_{\cdot,|1|}^{2}, x_{\cdot, 2 \mid 1}^{2}\right) \geq 3 .\end{cases}
$$

Now consider sequentially complete markets. Let $p_{\cdot \mid 1}^{*}=(1 / 2,1 / 2,1 / 2,1 / 2), q_{2 \mid 1}^{*}=$ $(1 / 2,1 / 2), p_{\cdot \mid 2}^{*}=(3 / 4,1 / 4)$. Consider the following allocation

$$
\begin{array}{ll}
x_{\mid 1}^{* 1}=(2,2,2,2) & x_{\cdot \mid 1}^{* 2}=(2,2,2,2) \\
x_{\mid 2}^{* 1}=(1,1) & x_{\mid \cdot 2}^{* 2}=(3,3)
\end{array}
$$

achieved by the asset allocations

$$
\begin{aligned}
& y_{2 \mid 1}^{* 1}=(0,4), \\
& y_{2 \mid 1}^{* 2}=(4,0) .
\end{aligned}
$$

It can be verified that $\left(p^{*}, q^{*}, x^{*}, y^{*}\right)$ is a naïve $\mathrm{SC}$ equilibrium. In particular it holds that the choice of $y_{2 \mid 1}^{* 1}=(0,4)$ and $y_{2 \mid 1}^{* 2}=(4,0)$ is optimal given prices and price expectations $p_{\cdot \mid 1}^{*}=(1 / 2,1 / 2,1 / 2,1 / 2)$ and it holds that for the subeconomy starting in period 2 with initial endowments $y_{2 \mid 1}^{* 1}=(0,4)$ and $y_{2 \mid 1}^{* 2}=(4,0)$, prices $p_{\cdot \mid 2}^{*}=(3 / 4,1 / 4)$ constitute a competitive equilibrium. The prices for contingent commodities in period 1 are not proportional to actual prices in period 2. Thus, households have incorrect expectations about prices. Since $x_{\cdot \mid 1}^{1}=(2,2,1,1)$ is not individually rational for household 1 from the perspective of period 1 , there can be no $p$ such that $(p,(2,2,1,1),(2,2,3,3))$ is an AD equilibrium.

When preferences are time-consistent, every $\mathrm{AD}$ equilibrium is allocationally equivalent to some naïve and to some sophisticated SC equilibrium. This is shown in the following theorems. 
Theorem 4.6 Let preferences be time-consistent and let the economy $\mathcal{E}$ be locally nonsatiated. Let $\left(p^{*}, x^{*}\right)$ be an $A D$ equilibrium of $\mathcal{E}$. Then there is $\left(q^{*}, y^{*}\right) \in \bar{Q} \times \bar{Y}$ such that $\left(p^{*}, q^{*}, x^{*}, y^{*}\right)$ is a naïve $S C$ equilibrium of $\mathcal{E}$.

Proof

Let $\left(p^{*}, x^{*}\right)$ be an $\mathrm{AD}$ equilibrium. We define $q_{\cdot \mid s(t)}^{*}=p_{t+1, T \mid s(t)}^{*}$ for every $s(t)$. By local non-satiation of the economy we know that for every $s, \sigma \in S, t, \tau \in T$ with $\tau>t$ and $\sigma(t)=s(t)$ there is an $l_{\sigma(\tau) \mid s(t)}$ with $p_{\sigma(\tau), l_{\sigma(\tau) \mid s(t)}^{*} \mid s(t)}^{*} \neq 0$. For every $s(t)$, we define $y_{\cdot \mid s(t)}^{* h}$ recursively as follows. Start by setting, for every $h \in H$ and every $\sigma \in S$ with $\sigma(t)=s(t)$,

$$
y_{\sigma(T), l_{\sigma(T) \mid s(t)}^{* h}(t)}^{* h}=\frac{p_{\sigma(T) \mid s(t)}^{*}\left(x_{\sigma(T) \mid s(t)}^{* h}-e_{\sigma(T) \mid s(t)}^{h}\right)}{p_{\sigma(T), l_{\sigma(T) \mid s(t)} \mid s(t)}^{*}}+e_{\sigma(T), l_{\sigma(T) \mid s(t)} \mid s(t)}^{h},
$$

and $y_{\sigma(T), l \mid s(t)}^{* h}=e_{\sigma(T), l \mid s(t)}^{h}$ for every $l \neq l_{\sigma(T) \mid s(t)}$. Then, continue by setting, for every $h \in H$, $t<\tau<T, \sigma \in S$ with $\sigma(t)=s(t)$,

$$
\begin{aligned}
y_{\sigma(\tau), l_{\sigma(\tau) \mid s(t)}^{* h}(t)}^{* h} & =\frac{p_{\sigma(\tau) \mid s(t)}^{*}\left(x_{\sigma(\tau) \mid s(t)}^{* h}-e_{\sigma(\tau) \mid s(t)}^{h}\right)}{p_{\sigma(\tau), l_{\sigma(\tau) \mid s(t)}^{*} \mid s(t)}^{*}}+e_{\sigma(\tau), l_{\sigma(\tau) \mid s(t)} \mid s(t)}^{h} \\
& +\frac{\sum_{\sigma(\tau+1) \in \sigma^{+}(\tau)}\left(p_{\sigma(\tau+1) \mid s(t)}^{*} y_{\sigma(\tau+1) \mid s(t)}^{* h}-p_{\sigma(\tau+1) \mid s(t)}^{*} e_{\sigma(\tau+1) \mid s(t)}^{h}\right)}{p_{\sigma(\tau), l_{\sigma(\tau) \mid s(t)} \mid s(t)}^{*}}
\end{aligned}
$$

and $y_{\sigma(\tau), l \mid s(t)}^{* h}=e_{\sigma(\tau), l \mid s(t)}^{h}$ for every $l \neq l_{\sigma(\tau) \mid s(t)}$.

Then it holds that

$$
p_{\sigma(T) \mid s(t)}^{*} y_{\sigma(T) \mid s(t)}^{* h}=p_{\sigma(T) \mid s(t)}^{*} x_{\sigma(T) \mid s(t)}^{* h}
$$

for all $s, \sigma, t, h$, and

$$
\begin{aligned}
& p_{\sigma(\tau) \mid s(t)}^{*} x_{\sigma(\tau) \mid s(t)}^{* h}+\sum_{\sigma(\tau+1) \in \sigma^{+}(\tau)} p_{\sigma(\tau+1) \mid s(t)}^{*} y_{\sigma(\tau+1) \mid s(t)}^{* h}= \\
& p_{\sigma(\tau) \mid s(t)}^{*} y_{\sigma(\tau) \mid s(t)}^{* h}+\sum_{\sigma(\tau+1) \in \sigma^{+}(\tau)} p_{\sigma(\tau+1) \mid s(t)}^{*} e_{\sigma(\tau+1) \mid s(t)}^{h} .
\end{aligned}
$$

for all $s, \sigma, h, t \leq \tau<T$.

We claim that $\left(p^{*}, q^{*}, x^{*}, y^{*}\right)$ is a naïve sequentially complete equilibrium.

By summing the budget constraints over all households, using that

$$
\sum_{h \in H} x_{\cdot \mid s(t)}^{* h}=\sum_{h \in H} e_{\cdot \mid s(t)}^{h},
$$

we obtain, for every $s, t$,

$$
\sum_{h \in H} y_{\cdot \mid s(t)}^{* h}=\sum_{h \in H} e_{\cdot \mid s(t+1)}^{h} .
$$


It therefore holds that conditions (b) and (c) of the definition of a naïve sequentially complete equilibrium are satisfied. It only remains to be checked that condition (a) is satisfied as well.

Consider any $t, s, h$, and any $\left(x_{\cdot \mid s(t)}^{h}, y_{\cdot \mid s(t)}^{h}\right) \in{ }^{n} \bar{\gamma}_{s(t)}^{h}\left(p_{\cdot \mid s(t)}^{*}, q_{\cdot \mid s(t)}^{*}, y_{s(t) \mid s(t-1)}^{* h}, e_{t+1, T \mid s(t)}^{h}\right)$. By summing up budget constraints, we have that

$$
\begin{aligned}
& p_{\cdot \mid s(t)}^{*} x_{\cdot \mid s(t)}^{h}+p_{t+1, T \mid s(t)}^{*} y_{t+1, T \mid s(t)}^{h} \leq \\
& p_{s(t) \mid s(t)}^{*} y_{s(t) \mid s(t-1)}^{* h}+p_{t+1, T \mid s(t)}^{*} e_{t+1, T \mid s(t)}^{h}+p_{t+1, T \mid s(t)}^{*} y_{t+1, T \mid s(t)}^{h},
\end{aligned}
$$

so,

$$
p_{\cdot \mid s(t)}^{*} x_{\cdot \mid s(t)}^{h} \leq p_{s(t) \mid s(t)}^{*} y_{s(t) \mid s(t-1)}^{* h}+p_{t+1, T \mid s(t)}^{*} e_{t+1, T \mid s(t)}^{h} .
$$

In addition, we have

$$
\begin{aligned}
p_{s(\tau) \mid s(\tau)}^{*} x_{s(\tau) \mid s(\tau)}^{* h}+\sum_{s(\tau+1) \in s^{+}(\tau)} p_{s(\tau+1) \mid s(\tau)}^{*} y_{s(\tau+1) \mid s(\tau)}^{* h} \leq \\
p_{s(\tau) \mid s(\tau)}^{*} y_{s(\tau) \mid s(\tau-1)}^{* h}+\sum_{s(\tau+1) \in s^{+}(\tau)} p_{s(\tau+1) \mid s(\tau)}^{*} e_{s(\tau+1) \mid s(\tau)}^{h}
\end{aligned}
$$

for every $\tau<t$.

By summing all these and by keeping in mind that $x^{*}$ is a time-consistent allocation, we find that $x_{\cdot \mid 1}^{* h} \prec x_{\cdot \mid s(t)}^{h} \in \breve{\gamma}_{1}^{h}\left(p_{\cdot \mid 1}^{*}, e_{\cdot \mid 1}^{h}\right)$.

Now suppose that there are $t, s, h$ with

$$
\left(x_{\cdot \mid s(t)}^{* h}, y_{\cdot \mid s(t)}^{* h}\right) \notin^{n} \bar{\delta}_{s(t)}^{h}\left(p_{\cdot \mid s(t)}^{*}, q_{\cdot \mid s(t)}^{*}, y_{s(t) \mid s(t-1)}^{* h}, e_{t+1, T \mid s(t)}^{h}\right) .
$$

Then there must be $\left(\hat{x}_{\cdot \mid s(t)}^{h}, \hat{y}_{\cdot \mid s(t)}^{h}\right) \in{ }^{n} \bar{\gamma}_{s(t)}^{h}\left(p_{\cdot \mid s(t)}^{*}, q_{\cdot \mid s(t)}^{*}, y_{s(t) \mid s(t-1)}^{* h}, e_{t+1, T \mid s(t)}^{h}\right)$ with $\hat{x}_{\cdot \mid s(t)}^{h} \succ^{h, s(t)}$ $x_{\cdot \mid s(t)}^{* h}$. But then, by time-consistency of preferences $x_{\cdot \mid 1}^{* h} \zeta \hat{x}_{\cdot \mid s(t)}^{h} \succ^{h, 1} x_{\cdot \mid 1}^{* h}$. By the discussion above we also have, $x_{\cdot \mid 1}^{* h} \zeta \hat{x}_{\cdot \mid s(t)}^{h} \in \breve{\gamma}_{1}^{h}\left(p_{\cdot \mid 1}^{*}, e_{\cdot \mid 1}^{h}\right)$. So $x_{\cdot \mid 1}^{* h} \notin \breve{\delta}_{1}^{h}\left(p_{\cdot \mid 1}^{*}, e_{\cdot \mid 1}^{h}\right)$, a contradiction to the fact that $\left(p^{*}, x^{*}\right)$ is an $\mathrm{AD}$ equilibrium.

Q.E.D.

Theorem 4.7 Let preferences be time-consistent and let the economy $\mathcal{E}$ be locally nonsatiated. Let $\left(p^{*}, x^{*}\right)$ be an $A D$ equilibrium of $\mathcal{E}$. Then there $i s\left(q^{*}, y^{*}\right) \in \bar{Q} \times \bar{Y}$ such that $\left(p^{*}, q^{*}, x^{*}, y^{*}\right)$ is a sophisticated $S C$ equilibrium of $\mathcal{E}$.

\section{Proof}

Define $q^{*}$ and $y^{*}$ as in Theorem 4.6. We only need to verify whether condition (a) of the definition of a sophisticated SC equilibrium is satisfied. By the same argument as in Theorem 4.6 we know that for every $s$

$$
x_{\cdot \mid s(T)}^{* h} \in{ }^{s} \bar{\delta}_{s(T)}^{h}\left(p_{\cdot \mid s(T)}^{*}, y_{s(T) \mid s(T-1)}^{* h}\right) .
$$

We continue the proof by an induction argument. Assume that, for some $t$,

$$
\left(x_{\cdot \mid s(\tau)}^{* h}, y_{\cdot \mid s(\tau)}^{* h}\right) \in \bar{\delta}_{s(\tau)}^{h}\left(p_{\cdot \mid s(\tau)}^{*}, q_{\cdot \mid s(\tau)}^{*}, y_{s(\tau) \mid s(\tau-1)}^{* h}, e_{\tau+1, T \mid s(\tau)}^{h}\right)
$$


for every $\tau>t$, for every $s$. Suppose that

$$
\left(x_{\cdot \mid s(t)}^{* h}, y_{\cdot \mid s(t)}^{* h}\right) \notin s \bar{\delta}_{s(t)}^{h}\left(p_{\cdot \mid s(t)}^{*}, q_{\cdot \mid s(t)}^{*}, y_{s(t) \mid s(t-1)}^{* h}, e_{t+1, T \mid s(t)}^{h}\right) .
$$

Then there must be $\left(\hat{x}_{\cdot \mid s(t)}^{h}, \hat{y}_{\cdot \mid s(t)}^{h}\right) \in{ }^{s} \bar{\gamma}_{s(t)}^{h}\left(p_{\cdot \mid s(t)}^{*}, q_{\cdot \mid s(t)}^{*}, y_{s(t) \mid s(t-1)}^{* h}, e_{t+1, T \mid s(t)}^{h}\right)$ with $\hat{x}_{\cdot \mid s(t)}^{h} \succ^{h, s(t)}$ $x_{\cdot \mid s(t)}^{* h}$. The same argument as in the proof of Theorem 4.6 then leads to a contradiction.

Q.E.D.

The following theorem shows that every sophisticated SC equilibrium is allocationally equivalent to some AD equilibrium if preferences are time-consistent. Notice that we need to make continuity assumptions for this direction.

Theorem 4.8 Let preferences be time-consistent and continuous, let consumption sets be closed and bounded from below, and let the economy $\mathcal{E}$ be locally non-satiated. Let $\left(p^{*}, q^{*}, x^{*}, y^{*}\right)$ be a sophisticated $S C$ equilibrium of $\mathcal{E}$ with $p^{*} \gg 0$. Then there is a $p$ such that $\left(p, x^{*}\right)$ is an $A D$ equilibrium of $\mathcal{E}$.

\section{Proof}

Let $\left(p^{*}, q^{*}, x^{*}, y^{*}\right)$ be a sophisticated $\mathrm{SC}$ equilibrium. By Theorem 4.4 , for every $s, \sigma \in S$ and every $t, \tau \in T$ with $\tau>t$ and $\sigma(t)=s(t)$, there must be $\mu_{\sigma(\tau) \mid s(t)}$ such that

$$
q_{\sigma(\tau) \mid s(t)}^{*}=\mu_{\sigma(\tau) \mid s(t)} p_{\sigma(\tau) \mid s(t)}^{*}
$$

Suppose that $\mu_{\sigma(\tau) \mid 1} \leq 0$ for some $\sigma(\tau)$. Then $q_{\sigma(\tau) \mid 1}^{*}=\mu_{\sigma(\tau) \mid 1} p_{\sigma(\tau) \mid 1}^{*} \leq 0$. Thus, it would be possible to increase available income in state $\sigma(\tau)$ without increasing expenditures in state $\sigma(\tau-1)$. A household $h$ that, from the perspective of period 1 , is locally non-satiated in state $\sigma(\tau)$, could change consumption in state $\sigma(\tau)$ and have a consumption plan that is strictly preferred to the current bundle. Since preferences are time-consistent, the new bundle would also be consistent with sophisticated behavior. This yields a contradiction against $x^{*}$ being an equilibrium allocation. It follows that $\mu_{\sigma(\tau) \mid 1}>0$ for every $\sigma(\tau)$.

Let $p_{1 \mid 1}=p_{1 \mid 1}^{*}$ and $p_{\sigma(\tau) \mid 1}=p_{\sigma(\tau) \mid 1}^{*} \prod_{\tau^{\prime}=2}^{\tau} \mu_{\sigma\left(\tau^{\prime}\right) \mid 1}$ for every $\sigma \in S$ and every $\tau>1$. Also, let $p_{\cdot \mid s(t)}=p_{s(t), T \mid 1}$ for all $t \in T, s \in S$. It holds that $p \gg 0$ and therefore that $\breve{\gamma}_{1}^{h}\left(p_{\cdot \mid 1}, e_{\cdot \mid 1}^{h}\right)$ is compact.

Suppose that $\left(p, x^{*}\right)$ is not an AD equilibrium. Then there is $s(t)$ with $x_{\cdot \mid s(t)}^{* h} \notin$ $\breve{\delta}_{s(t)}^{h}\left(p_{\cdot \mid s(t)}, x_{s(t), T \mid s(t-1)}^{* h}\right)$. Since we have $x_{\cdot \mid s(t)}^{* h}=x_{s(t), T \mid s(t-1)}^{* h}$ for every $t>1$, it should hold that $s(t)=1$. We know that $x_{\cdot \mid 1}^{* h} \in \breve{\gamma}_{1}^{h}\left(p_{\cdot \mid 1}, e_{\cdot \mid 1}^{h}\right)$. So there is $\bar{x}_{\cdot \mid 1}^{h}$ with $\bar{x}_{\cdot \mid 1}^{h} \in \breve{\gamma}_{1}^{h}\left(p_{\cdot \mid 1}, e_{\cdot \mid 1}^{h}\right)$ and $\bar{x}_{\cdot \mid 1}^{h} \succ^{h, 1} x_{\cdot \mid 1}^{* h}$. Since $\succeq^{h, 1}$ is continuous and $\breve{\gamma}_{1}^{h}\left(p_{\cdot \mid 1}, e_{\cdot \mid 1}^{h}\right)$ is compact, there exists a maximum on $\breve{\gamma}_{1}^{h}\left(p_{\cdot \mid 1}, e_{\cdot \mid 1}^{h}\right)$ with respect to $\succeq^{h, 1}$. Without loss of generality, $\bar{x}_{\cdot \mid 1}^{h}$ equals that maximum. We define $\bar{x}_{\cdot \mid s(t)}^{h}=\bar{x}_{s(t), T \mid 1}^{h}$ for every $s(t)$. By the same argument as in the proof of Theorem 4.6, there must be $\bar{y}$ with $\left(\bar{x}_{\cdot \mid 1}^{h}, \bar{y}_{\cdot \mid 1}^{h}\right) \in{ }^{n} \bar{\gamma}_{1}^{h}\left(p_{\cdot \mid 1}, p_{2, T \mid 1}, e_{\cdot \mid 1}^{h}\right)$ and $\bar{y}_{s(t+1), T \mid s(t)}^{h}=\bar{y}_{s(t+1), T \mid 1}^{h}$ for every $s(t)$. Moreover, from the opportunity sets, it follows that $n \bar{\gamma}_{1}^{h}\left(p_{\cdot \mid 1}, p_{2, T \mid 1}, e_{\cdot \mid 1}^{h}\right)={ }^{n} \bar{\gamma}_{1}^{h}\left(p_{\cdot \mid 1}^{*}, q_{\cdot \mid 1}^{*}, e_{\cdot \mid 1}^{h}\right)$. 
Then, by definition of $\bar{x}_{\cdot \mid 1}^{h}$ and the fact that $\bar{x}_{\cdot \mid 1}^{h} \succ^{h, 1} x_{\cdot \mid 1}^{* h}$, there is $s(t)$ such that

$$
\left(\bar{x}_{\cdot \mid s(t)}^{h}, y_{\cdot \mid s(t)}^{h}\right) \notin s \bar{\delta}_{s(t)}^{h}\left(p_{\cdot \mid s(t)}^{*}, q_{\cdot \mid s(t)}^{*}, \bar{y}_{s(t) \mid s(t-1)}^{h}, e_{t+1, T \mid s(t)}^{h}\right)
$$

for any choice of $y_{\cdot \mid s(t)}^{h}$. Two cases can be distinguished.

Case 1:

There exists $y_{\cdot \mid s(t)}^{h}$ with $\left(\bar{x}_{\cdot \mid s(t)}^{h}, y_{\cdot \mid s(t)}^{h}\right) \in{ }^{s} \bar{\gamma}_{s(t)}^{h}\left(p_{\cdot \mid s(t)}^{*}, q_{\cdot \mid s(t)}^{*}, \bar{y}_{s(t) \mid s(t-1)}^{h}, e_{t+1, T \mid s(t)}^{h}\right)$. Then there must be $\left(\hat{x}_{\cdot \mid s(t)}^{h}, \tilde{y}_{\cdot \mid s(t)}^{h}\right) \in s \bar{\gamma}_{s(t)}^{h}\left(p_{\cdot \mid s(t)}^{*}, q_{\cdot \mid s(t)}^{*}, \bar{y}_{s(t) \mid s(t-1)}^{h}, e_{t+1, T \mid s(t)}^{h}\right)$ with $\hat{x}_{\cdot \mid s(t)}^{h} \succ^{h, s(t)} \bar{x}_{\cdot \mid s(t)}^{h}$. By time-consistency of preferences, it follows that $\bar{x}_{\cdot \mid 1}^{h} \prec \hat{x}_{\cdot \mid s(t)}^{h} \succ^{h, 1} \bar{x}_{\cdot \mid 1}^{h}$. Also, it can be checked that $\bar{x}_{\cdot \mid 1}^{h} \prec \hat{x}_{\cdot \mid s(t)}^{h} \in \breve{\gamma}_{1}^{h}\left(p_{\cdot \mid 1}, e_{\cdot \mid 1}^{h}\right)$. This contradicts the definition of $\bar{x}_{\cdot \mid 1}^{h}$. Thus, case 1 is not possible.

Case 2:

There does not exist $y_{\cdot \mid s(t)}^{h}$ with $\left(\bar{x}_{\cdot \mid s(t)}^{h}, y_{\cdot \mid s(t)}^{h}\right) \in{ }^{s} \bar{\gamma}_{s(t)}^{h}\left(p_{\cdot \mid s(t)}^{*}, q_{\cdot \mid s(t)}^{*}, \bar{y}_{s(t) \mid s(t-1)}^{h}, e_{t+1, T \mid s(t)}^{h}\right)$. Since $\bar{x}_{\cdot \mid 1}^{h} \in \breve{\gamma}_{1}^{h}\left(p_{\cdot \mid 1}, e_{\cdot \mid 1}^{h}\right)$ and relative prices do not change over time and are correctly anticipated, this can only happen if there is $s\left(t^{\prime}\right)$ with $t^{\prime}>t$ and

$$
\left(\bar{x}_{\cdot \mid s\left(t^{\prime}\right)}^{h}, \tilde{y}_{\cdot \mid s\left(t^{\prime}\right)}^{h}\right) \notin s \bar{\delta}_{s\left(t^{\prime}\right)}^{h}\left(p_{\cdot \mid s\left(t^{\prime}\right)}^{*}, q_{\cdot \mid s\left(t^{\prime}\right)}^{*}, \bar{y}_{s\left(t^{\prime}\right) \mid s\left(t^{\prime}-1\right)}^{h}, e_{t^{\prime}+1, T \mid s\left(t^{\prime}\right)}^{h}\right)
$$

for every $\tilde{y}_{\cdot \mid s\left(t^{\prime}\right)}^{h}$.

Next, as before, for $s\left(t^{\prime}\right)$ we can distinguish two cases. We repeat this reasoning until we end up at the last period. For the last period, we can no longer distinguish two cases. In the last period, only case 1 can happen. This again leads to a contradiction by a similar reasoning as before.

Q.E.D.

In general, for the time-inconsistent case, there is no relation between AD equilibria and naïve or sophisticated SC equilibria. When preferences are time-inconsistent conflicts arise between current and future preferences. A naïve household will typically deviate from planned consumption, since he does not anticipate a change in his future preferences. Sophisticated households do anticipate future changes, and are therefore constrained by their future behavior. On the contrary, the AD market setting ensures perfect commitment in period 1, which is optimal from the perspective of the preferences of households in period 1. Constraints coming from the behavior of future selves are thereby irrelevant.

\section{Complete Markets}

In this section there are complete markets for all contingent commodities at every event of every period. Again, $p_{\cdot \mid s(t)}$ denotes the expected (at state $s(t)$ ) prices on the spot markets. In state $s(t)$, the expected state $\sigma(\tau)$ prices for contingent commodities that are delivered in state $\tilde{\sigma}(\tilde{\tau})$ are given by $q_{(\tilde{\sigma}(\tilde{\tau}) \mid \sigma(\tau)) \mid s(t)}$, where $\tilde{\tau}>\tau \geq t, \tilde{\sigma}(\tau)=\sigma(\tau)$, and $\sigma(t)=s(t)$. The expectations in state $s(t)$ of prices for all one-period ahead contingent commodities that can possibly be traded from state $\sigma(\tau)$ on, are denoted by $q_{(\cdot \mid \sigma(\tau), T) \mid s(t)}$. We define $Q_{\cdot \mid s(t)}=\mathbb{R}^{\sum_{\tilde{\tau}=t}^{T-1} \mathcal{L}_{\tilde{\tau}}}$. We let $y_{(\sigma(\tilde{\tau}) \mid \sigma(\tau)) \mid s(t)}^{h}$ denote bundles of contingent commodities that 
are expected at $s(t)$ to be bought in $\sigma(\tau)$ and that are delivered in $\sigma(\tilde{\tau})$. For notational purposes, we define $y_{(\cdot \mid s(0)) \mid s(0)}^{h}=e_{\cdot \mid 1}^{h}$. We also define $Y_{\cdot \mid s(t)}^{h}=\mathbb{R}^{\sum_{\tilde{\tau}=t}^{T-1} \mathcal{L}_{\tilde{\tau}}}$. Finally,

$$
X=\prod_{h \in H} \prod_{s(t)} X_{\cdot \mid s(t)}^{h}, \quad Y=\prod_{h \in H} \prod_{s(t)} Y_{\cdot \mid s(t)}^{h}, \quad P=\prod_{s(t)} P_{\cdot \mid s(t)}, \quad Q=\prod_{s(t)} Q_{\cdot \mid s(t)}
$$

\subsection{Naïve Behavior}

In every state, households sell their endowments which are determined by previous purchases of contingent commodities. With the revenues of these sales they buy goods on the spot markets and contingent commodities on asset markets. The contingent commodities bought determine the endowments at future states. In order to make a choice, a household needs to have expectations about prices of goods and contingent commodities in the future so as to know what to buy. The opportunity set of household $h$ in state $s(t)$ is given by

$$
\begin{array}{r}
{ }^{n} \gamma_{s(t)}^{h}\left(p_{\cdot \mid s(t)}, q_{\cdot \mid s(t)}, y_{(\cdot \mid s(t-1)) \mid s(t-1)}^{h}\right)=\left\{\left(x_{\cdot \mid s(t)}^{h}, y_{\cdot \mid s(t)}^{h}\right) \in X_{\cdot \mid s(t)}^{h} \times Y_{\cdot \mid s(t)}^{h} \mid\right. \\
p_{\sigma(\tau) \mid s(t)} x_{\sigma(\tau) \mid s(t)}^{h}+q_{(\cdot \mid \sigma(\tau)) \mid s(t)} y_{(\cdot \mid \sigma(\tau)) \mid s(t)}^{h} \leq \\
p_{\sigma(\tau) \mid s(t)} y_{(\sigma(\tau) \mid \sigma(\tau-1)) \mid s(t)}^{h}+q_{(\cdot \mid \sigma(\tau)) \mid s(t)} y_{\left(\sigma^{+}(\tau), T \mid \sigma(\tau-1)\right) \mid s(t)}^{h}
\end{array}
$$

for every $\tau \geq t$, and every $\sigma(\tau)$ with $\sigma(t)=s(t)\}$,

where, by definition, $y_{(\cdot \mid 0) \mid 0}^{h}=e_{\cdot \mid 1}^{h}$ and $y_{(\cdot \mid s(t-1)) \mid s(t)}^{h}=y_{(\cdot \mid s(t-1)) \mid s(t-1)}^{h}$. The demand set is then given by

$$
\begin{array}{r}
{ }^{n} \delta_{s(t)}^{h}\left(p_{\cdot \mid s(t)}, q_{\cdot \mid s(t)}, y_{(\cdot \mid s(t-1)) \mid s(t-1)}^{h}\right)=\left\{\left(\bar{x}_{\cdot \mid s(t)}^{h}, \bar{y}_{\cdot \mid s(t)}^{h}\right) \in{ }^{n} \gamma_{s(t)}^{h}\left(p_{\cdot \mid s(t)}, q_{\cdot \mid s(t)}, y_{(\cdot \mid s(t-1)) \mid s(t-1)}^{h}\right) \mid\right. \\
\left.\nexists\left(x_{\cdot \mid s(t)}^{h}, y_{\cdot \mid s(t)}^{h}\right) \in{ }^{n} \gamma_{s(t)}^{h}\left(p_{\cdot \mid s(t)}, q_{\cdot \mid s(t)}, y_{(\cdot \mid s(t-1)) \mid s(t-1)}^{h}\right) \text { with } x_{\cdot \mid s(t)}^{h} \succ^{h, s(t)} \bar{x}_{\cdot \mid s(t)}^{h}\right\} .
\end{array}
$$

In equilibrium all markets should clear and should be expected to clear.

\section{Definition 5.1 Naïve Complete Equilibrium}

A pair $\left(p^{*}, q^{*}, x^{*}, y^{*}\right) \in P \times Q \times X \times Y$ is a Naïve Complete equilibrium of the economy $\mathcal{E}$ if

(a) $\left(x_{\cdot \mid s(t)}^{* h}, y_{\cdot \mid s(t)}^{* h}\right) \in{ }^{n} \delta_{s(t)}^{h}\left(p_{\cdot \mid s(t)}^{*}, q_{\cdot \mid s(t)}^{*}, y_{(\cdot \mid s(t-1)) \mid s(t-1)}^{* h}\right)$ for all $h \in H, t \in T, s \in S$,

(b) $\sum_{h \in H} x_{\cdot \mid s(t)}^{* h}=\sum_{h \in H} e_{\cdot \mid s(t)}^{h}$ for all $t \in T, s \in S$, and

(c) $\sum_{h \in H} y_{(\cdot \mid s(\tau)) \mid s(t)}^{* h}=\sum_{h \in H} e_{\tau+1, T \mid s(\tau)}^{h}$ for all $t, \tau \in T, s \in S$.

\subsection{Sophisticated Behavior}

In period $T$ the opportunity set of a sophisticated household $h$ in state $s$ is the same as the opportunity set for its naïve counterpart, i.e.

$$
{ }^{s} \gamma_{s(T)}^{h}\left(p_{\cdot \mid s(T)}, y_{\cdot \mid s(T-1)}^{h}\right)={ }^{n} \gamma_{s(T)}^{h}\left(p_{\cdot \mid s(T)}, y_{\cdot \mid s(T-1)}^{h}\right)
$$


The opportunity set of a sophisticated household $h$ in state $s(t)$, where $t<T$, is given by

$$
{ }^{s} \gamma_{s(t)}^{h}\left(p_{\cdot \mid s(t)}, q \cdot \mid s(t), y_{(\cdot \mid s(t-1)) \mid s(t-1)}^{h}\right)=\left\{\left(x_{\cdot \mid s(t)}^{h}, y_{\cdot \mid s(t)}^{h}\right) \in X_{\cdot \mid s(t)}^{h} \times Y_{\cdot \mid s(t)}^{h} \mid\right.
$$

(i) $p_{\sigma(\tau) \mid s(t)} x_{\sigma(\tau) \mid s(t)}^{h}+q_{(\cdot \mid \sigma(\tau)) \mid s(t)} y_{(\cdot \mid \sigma(\tau)) \mid s(t)}^{h} \leq$

$$
\begin{aligned}
& p_{\sigma(\tau) \mid s(t)} y_{(\sigma(\tau) \mid \sigma(\tau-1)) \mid s(t)}^{h}+q_{(\cdot \mid \sigma(\tau)) \mid s(t)} y_{\left(\sigma^{+}(\tau), T \mid \sigma(\tau-1)\right) \mid s(t)}^{h} \\
& \text { for every } \tau \geq t, \text { and every } \sigma(\tau) \text { with } \sigma(t)=s(t),
\end{aligned}
$$

and

(ii) $\left(x_{s(t+1), T \mid s(t)}^{h}, y_{(\cdot \mid s(t+1), T) \mid s(t)}^{h}\right) \in$

$$
s \delta_{s(t+1)}^{h}\left(p_{s(t+1), T \mid s(t)}, q_{(\cdot \mid s(t+1), T) \mid s(t)}, y_{(\cdot \mid s(t)) \mid s(t)}^{h}\right)
$$

$$
\text { for every } \left.s(t+1) \in s^{+}(t)\right\} \text {. }
$$

The demand set of a sophisticated household is as follows.

$$
\begin{aligned}
{ }^{s} \delta_{s(t)}^{h}\left(p_{\cdot \mid s(t)}, q_{\cdot \mid s(t)}, y_{(\cdot \mid s(t-1)) \mid s(t-1)}^{h}\right)=\left\{\left(\bar{x}_{\cdot \mid s(t)}^{h}, \bar{y}_{\cdot \mid s(t)}^{h}\right) \in{ }^{s} \gamma_{s(t)}^{h}\left(p_{\cdot \mid s(t)}, q_{\cdot \mid s(t)}, y_{(\cdot \mid s(t-1)) \mid s(t-1)}^{h}\right) \mid\right. \\
\\
\left.\nexists\left(x_{\cdot \mid s(t)}^{h}, y_{\cdot \mid s(t)}^{h}\right) \in{ }^{s} \gamma_{s(t)}^{h}\left(p_{\cdot \mid s(t)}, q_{\cdot \mid s(t)}, y_{(\cdot \mid s(t-1)) \mid s(t-1)}^{h}\right) \text { with } x_{\cdot \mid s(t)}^{h} \succ^{h, s(t)} \bar{x}_{\cdot \mid s(t)}^{h}\right\} .
\end{aligned}
$$

Finally, we define a sophisticated complete equilibrium as follows.

\section{Definition 5.2 Sophisticated Complete Equilibrium}

A pair $\left(p^{*}, q^{*}, x^{*}, y^{*}\right) \in P \times Q \times X \times Y$ is a Sophisticated Complete equilibrium of the economy $\mathcal{E}$ if

(a) $\left(x_{\cdot \mid s(t)}^{* h}, y_{\cdot \mid s(t)}^{* h}\right) \in{ }^{s} \delta_{s(t)}^{h}\left(p_{\cdot \mid s(t)}^{*}, q_{\cdot \mid s(t)}^{*}, y_{(\cdot \mid s(t-1)) \mid s(t-1)}^{* h}\right)$ for all $h \in H, t \in T, s \in S$,

(b) $\sum_{h \in H} x_{\cdot \mid s(t)}^{* h}=\sum_{h \in H} e_{\cdot \mid s(t)}^{h}$ for all $t \in T, s \in S$,

(c) $\sum_{h \in H} y_{(\cdot \mid s(\tau)) \mid s(t)}^{* h}=\sum_{h \in H} e_{\tau+1, T \mid s(\tau)}^{h}$ for all $t, \tau \in T, s \in S$,

(d) $p_{s(\tau), T \mid s(t)}^{*}=p_{\cdot \mid s(\tau)}^{*}$ for all $s \in S, t, \tau \in T$ with $t \leq \tau$,

(e) $q_{(\cdot \mid s(\tau)) \mid s(t)}^{*}=q_{\cdot \mid s(\tau)}^{*}$ for all $s \in S, t, \tau \in T$ with $t \leq \tau$,

(f) $x_{s(\tau), T \mid s(t)}^{* h}=x_{\cdot s(\tau)}^{* h}$ for all $h \in H, s \in S, t, \tau \in T$ with $t \leq \tau$, and

(g) $y_{(\cdot \mid s(\tau)) \mid s(t)}^{* h}=y_{\cdot \mid s(\tau)}^{* h}$ for all $h \in H, s \in S, t, \tau \in T$ with $t \leq \tau$.

\subsection{Properties of Complete Equilibria}

An $\mathrm{AD}$ equilibrium $\left(p^{*}, q^{*}\right)$ is said to be allocationally equivalent to a naïve (sophisticated) complete equilibrium if there are $p \in P, q \in Q$ and $y \in Y$ such that $\left(p, q, x^{*}, y\right)$ is a naïve (sophisticated) complete equilibrium. Similarly, a naïve (sophisticated) complete equilibrium $\left(p^{*}, q^{*}, x^{*}, y^{*}\right)$ is said to be allocationally equivalent to an $\mathrm{AD}$ equilibrium if 
there is a $p \in P$ such that $\left(p, x^{*}\right)$ is an $\mathrm{AD}$ equilibrium. A naïve (sophisticated) complete equilibrium $\left(p^{*}, q^{*}, x^{*}, y^{*}\right)$ is said to be allocationally equivalent to a naïve (sophisticated) SC equilibrium if there are $p \in P, q \in \bar{Q}$ and $y \in \bar{Y}$ such that $\left(p, q, x^{*}, y\right)$ is a naïve (sophisticated) SC equilibrium. A naïve (sophisticated) $\mathrm{SC}$ equilibrium $\left(p^{*}, q^{*}, x^{*}, y^{*}\right)$ is said to be allocationally equivalent to a naïve (sophisticated) complete equilibrium if there are $p \in P, q \in Q$ and $y \in Y$ such that $\left(p, q, x^{*}, y\right)$ is a naïve (sophisticated) complete equilibrium.

In complete equilibria, relative prices are expected to remain unchanged. This is proven in the following theorem.

Theorem 5.3 Let the economy $\mathcal{E}$ be locally non-satiated and let $\left(p^{*}, q^{*}, x^{*}, y^{*}\right)$ be a naïve (sophisticated) complete equilibrium of $\mathcal{E}$. Then for every $s, \sigma$ and every $t, \tau$ with $t<\tau$ there must be $\mu_{\sigma(\tau) \mid s(t)} \in \mathbb{R}$ with

$$
q_{(\sigma(\tau), T \mid \sigma(\tau-1)) \mid s(t)}^{*}=\mu_{\sigma(\tau) \mid s(t)}\left(p_{\sigma(\tau) \mid s(t)}^{*}, q_{(\cdot \mid \sigma(\tau)) \mid s(t)}^{*}\right) .
$$

\section{Proof}

Consider some $s, \sigma \in S$ and $t, \tau \in T$ with $\tau>t$ and $\sigma(t)=s(t)$. Since the economy is locally non-satiated, it holds that $p_{\sigma(\tau) \mid s(t)}^{*} \neq 0$. Let $l$ be a commodity such that $p_{\sigma(\tau), l \mid s(t)}^{*} \neq 0$.

Apart from the fact that we have more cases, the remainder of the proof is analogous to the proof of Theorem 4.3. Suppose that there is no $\mu \in \mathbb{R}$ with both

$$
\begin{aligned}
& \mu p_{\sigma(\tau), l \mid s(t)}^{*}=q_{(\sigma(\tau), l \mid \sigma(\tau-1)) \mid s(t)}^{*}, \\
& \mu p_{\sigma(\tau), \tilde{l} \mid s(t)}^{*}=q_{(\sigma(\tau), \tilde{l} \mid \sigma(\tau-1)) \mid s(t)}^{*}
\end{aligned}
$$

or that there is no $\mu$ with both

$$
\begin{aligned}
\mu p_{\sigma(\tau), l \mid s(t)}^{*} & =q_{(\sigma(\tau), l \mid \sigma(\tau-1)) \mid s(t)}^{*} \text { and } \\
\mu q_{(\tilde{\sigma}(\tilde{\tau}), \tilde{l} \mid \sigma(\tau)) \mid s(t)}^{*} & =q_{(\tilde{\sigma}(\tilde{\tau}), \tilde{l} \mid \sigma(\tau-1)) \mid s(t)}^{*} .
\end{aligned}
$$

Next a contradiction can be obtained and the proof can be finished in a similar way as in the proof of Theorem 4.3.

Q.E.D.

In general, when preferences are allowed to be time-inconsistent, there is no link between naïve (sophisticated) complete equilibria and $\mathrm{AD}$ equilibria. In $\mathrm{AD}$ equilibria, because of the commitment power provided by the AD market structure, future selves of households have no influence. In naïve complete equilibria, future selves will typically deviate from the plans of current selves. In sophisticated complete equilibria, current selves are constrained by the optimizing behavior of future selves.

When preferences are time-consistent, every $\mathrm{AD}$ equilibrium is allocationally equivalent to some naïve (sophisticated) complete equilibrium. This is shown in the following two theorems.

Theorem 5.4 Let preferences in the economy $\mathcal{E}$ be time-consistent and let $\left(p^{*}, x^{*}\right)$ be an $A D$ equilibrium of $\mathcal{E}$. Then $\left(p^{*}, q^{*}, x^{*}, y^{*}\right)$ is a naïve complete equilibrium of $\mathcal{E}$, where 
$y_{(\sigma(\tilde{\tau}) \mid \sigma(\tau)) \mid s(t)}^{* h}=x_{\sigma(\tilde{\tau}) \mid s(t)}^{* h}$ for every $h, \sigma, s, \tilde{\tau}>\tau \geq t$ and $q_{(\sigma(\tilde{\tau}) \mid \sigma(\tau)) \mid s(t)}^{*}=p_{\sigma(\tilde{\tau}) \mid s(t)}^{*}$ for every $\sigma, s, \tilde{\tau}>\tau \geq t$.

\section{Proof}

Let prices be as described in the theorem. First of all, we need one observation. Consider

$$
\left(x_{\cdot \mid s(t)}^{h}, y_{\cdot \mid s(t)}^{h}\right) \in{ }^{n} \gamma_{s(t)}^{h}\left(p_{\cdot \mid s(t)}^{*}, q_{\cdot \mid s(t)}^{*}, y_{(\cdot \mid s(t-1)) \mid s(t-1)}^{* h}\right)
$$

Then we have

$$
p_{\sigma(\tau) \mid s(t)}^{*} x_{\sigma(\tau) \mid s(t)}^{h}+p_{\sigma^{+}(\tau), T \mid s(\tau)}^{*} y_{(\tau+1, T \mid \sigma(\tau)) \mid s(t)}^{h} \leq p_{\sigma(\tau), T \mid s(t)}^{*} y_{(\sigma(\tau), T \mid \sigma(\tau-1)) \mid s(t)}^{h}
$$

for every $\sigma, \tau \geq t$. By adding these over all $\sigma(\tau)$ with $\tau \geq t$ and $\sigma(t)=s(t)$, we get

$$
p_{\cdot \mid s(t)}^{*} x_{\cdot \mid s(t)}^{h} \leq p_{\cdot \mid s(t)}^{*} y_{(s(t), T \mid s(t-1)) \mid s(t-1)}^{h} \cdot
$$

Thus, we also have

$$
p_{\cdot \mid \sigma(t)}^{*} x_{\cdot \mid \sigma(t)}^{* h} \leq p_{\cdot \mid \sigma(t)}^{*} y_{(\sigma(t-1), T \mid \sigma(t-1)) \mid \sigma(t)}^{* h} .
$$

for every $\sigma$. Similarly, for all $\tau<t$, we know that

$$
p_{s(\tau) \mid s(\tau)}^{*} x_{s(\tau) \mid s(\tau)}^{* h}+p_{\tau+1, T \mid s(\tau)}^{*} y_{(\tau+1, T \mid s(\tau)) \mid s(\tau)}^{* h} \leq p_{\cdot \mid s(\tau)}^{*} y_{(s(\tau), T \mid s(\tau-1)) \mid s(\tau-1)}^{* h},
$$

by definition of $y^{*}$. Using that $p_{s(\tau), T \mid s(t)}^{*}=p_{\cdot \mid s(\tau)}^{*}$, and taking the sum of equation (2) for all $\sigma(t) \neq s(t)$, equation (1) and equation (3) for all $s(\tau)$ with $\tau<t$, we obtain $x_{\cdot \mid 1}^{* h} \prec x_{\cdot \mid s(t)}^{h} \in \breve{\gamma}_{1}^{h}\left(p_{\cdot \mid 1}^{*}, e_{\cdot \mid 1}^{h}\right)$.

With this observation in mind, we can continue the proof. Since $\left(p^{*}, x^{*}\right)$ is an $\mathrm{AD}$ equilibrium and by definition of $y^{*}$, it follows immediately that conditions (b) and (c) of the definition of a naïve complete equilibrium are satisfied. It remains to be shown that condition (a) is satisfied as well. Suppose there are $t, s(t), h$ with $\left(x_{\cdot \mid s(t)}^{* h}, y_{\cdot \mid s(t)}^{* h}\right) \notin$ ${ }^{n} \delta_{s(t)}^{h}\left(p_{\cdot \mid s(t)}^{*}, q_{\cdot \mid s(t)}^{*}, y_{\cdot \cdot s(t-1)) \mid s(t-1)}^{* h}\right)$. Then there must be

$$
\left(\hat{x}_{\cdot \mid s(t)}^{h}, y_{\cdot \mid s(t)}^{h}\right) \in{ }^{n} \gamma_{s(t)}^{h}\left(p_{\cdot \mid s(t)}^{*}, q_{\cdot \mid s(t)}^{*}, y_{(\cdot \mid s(t-1)) \mid s(t-1)}^{* h}\right)
$$

with $\hat{x}_{\cdot \mid s(t)}^{h} \succ^{h, s(t)} x_{\cdot \mid s(t)}^{* h}$. But then, by time-consistency of preferences, $x_{\cdot \mid 1}^{* h} \hat{x}_{\cdot \mid s(t)}^{h} \succ^{h, 1} x_{\cdot \mid 1}^{* h}$. By our observation, it follows that $x_{\cdot \mid 1}^{* h}\left\langle\hat{x}_{\cdot \mid s(t)}^{h} \in \breve{\gamma}_{1}^{h}\left(p_{\cdot \mid 1}^{*}, e_{\cdot \mid 1}^{h}\right)\right.$. So $x_{\cdot \mid 1}^{* h} \notin \breve{\delta}_{1}^{h}\left(p_{\cdot \mid 1}^{*}, e_{\cdot \mid 1}^{h}\right)$, contradicting that $\left(p^{*}, x^{*}\right)$ is an $\mathrm{AD}$ equilibrium.

Q.E.D.

Theorem 5.5 Let preferences in the economy $\mathcal{E}$ be time-consistent and let $\left(p^{*}, x^{*}\right)$ be an $A D$ equilibrium of $\mathcal{E}$. Then $\left(p^{*}, q^{*}, x^{*}, y^{*}\right)$ is a sophisticated complete equilibrium of $\mathcal{E}$, where $y_{(\sigma(\tilde{\tau}) \mid \sigma(\tau)) \mid s(t)}^{* h}=x_{\sigma(\tilde{\tau}) \mid s(t)}^{* h}$ for every $h, \sigma, s, \tilde{\tau}>\tau \geq t$ and $q_{(\sigma(\tilde{\tau}) \mid \sigma(\tau)) \mid s(t)}^{*}=p_{\sigma(\tilde{\tau}) \mid s(t)}^{*}$ for every $\sigma, s, \tilde{\tau}>\tau \geq t$.

Proof 
First, of all, by definition of $p^{*}, q^{*}, x^{*}$, and $y^{*}$, it follows immediately that conditions (b), (c), (d), (e), (f), and (g) of the definition of a sophisticated complete equilibrium are satisfied. It remains to be shown that condition (a) is satisfied as well.

By the same argument as used in the proof of Theorem 5.4, it holds that

$$
\left(x_{\cdot \mid s(T)}^{* h}, y_{\cdot \mid s(T)}^{* h}\right) \in{ }^{s} \delta_{s(T)}^{h}\left(p_{\cdot \mid s(T)}^{*}, y_{\cdot \mid s(T-1)}^{* h}\right) .
$$

We proceed with an induction argument. Let $s \in S, t \in T$. Assume that

$$
\left(x_{\cdot \mid s(t+1)}^{* h}, y_{\cdot \mid s(t+1)}^{* h}\right) \in{ }^{s} \delta_{s(t+1)}^{h}\left(p_{\cdot \mid s(t+1)}^{*}, q_{\cdot \mid s(t+1)}^{*}, y_{(\cdot \mid s(t)) \mid s(t)}^{* h}\right)
$$

for every $s(t+1) \in s^{+}(t)$. Suppose that $\left(x_{\cdot \mid s(t)}^{* h}, y_{\cdot \mid s(t)}^{* h}\right) \notin s \delta_{s(t)}^{h}\left(p_{\cdot \mid s(t)}^{*}, q_{\cdot \mid s(t)}^{*}, y_{\cdot(s(t-1)) \mid s(t-1)}^{* h}\right)$. Then there must be $\left(\hat{x}_{\cdot \mid s(t)}^{h}, \hat{y}_{\cdot \mid s(t)}^{h}\right) \in{ }^{s} \gamma_{s(t)}^{h}\left(p_{\cdot \mid s(t)}^{*}, q_{\cdot \mid s(t)}^{*}, y_{(\cdot \mid s(t-1)) \mid s(t-1)}^{* h}\right)$ with $\hat{x}_{\cdot \mid s(t)}^{h} \succ^{h, s(t)}$ $x_{\cdot \mid s(t)}^{* h}$. But then, by time-consistency of preferences $x_{\cdot \mid 1}^{* h} \zeta \hat{x}_{\cdot \mid s(t)}^{h} \succ^{h, 1} x_{\cdot \mid 1}^{* h}$. By the same argument as in the proof of Theorem 5.4 we also have $x_{\cdot \mid 1}^{* h} \prec \hat{x}_{\cdot \mid s(t)}^{h} \in \breve{\gamma}_{1}^{h}\left(p_{\cdot \mid 1}^{*}, e_{\cdot \mid 1}^{h}\right)$. This contradicts $\left(p^{*}, x^{*}\right)$ being an $\mathrm{AD}$ equilibrium.

Q.E.D.

The following example shows that even when preferences are time-consistent, not every naïve complete equilibrium is allocationally equivalent to an AD equilibrium.

Example 5.6 Consider Example 4.5. For two-period economies, the sequentially complete market structure is identical to the complete market structure. Thus, $\left(p^{*}, q^{*}, x^{*}, y^{*}\right)$ is also a naïve complete equilibrium. Again, there is no $p$ such that $(p,(2,2,1,1),(2,2,3,3))$ is an AD equilibrium.

Every sophisticated complete equilibrium, however, is allocationally equivalent to some AD equilibrium when preferences are time-consistent. Again, we need to make continuity assumptions.

Theorem 5.7 Let preferences be time-consistent and continuous, let consumption sets be closed and bounded from below, and let the economy $\mathcal{E}$ be locally non-satiated. Let $\left(p^{*}, q^{*}, x^{*}, y^{*}\right)$ be a sophisticated complete equilibrium of $\mathcal{E}$ with $p^{*} \gg 0$. Then $\left(p, x^{*}\right)$ is an $A D$ equilibrium of $\mathcal{E}$, where $p_{1 \mid 1}=p_{1 \mid 1}^{*}$ and $p_{2, T \mid 1}=q_{2, T|1| 1}^{*}$.

Proof

This proof is analogous to the proof for the sophisticated SC equilibrium in Theorem 4.8.

Q.E.D.

So far we have studied the relation between AD equilibria and SC and complete equilibria, respectively. Now we will analyze the relation between the latter two. The next theorem shows that every naïve SC equilibrium is allocationally equivalent to some naïve complete equilibrium. Notice that there is no need to assume time-consistency to obtain this result. 
Theorem 5.8 Let the economy $\mathcal{E}$ be locally non-satiated and let $\left(p^{*}, q^{*}, x^{*}, y^{*}\right)$ be a naïve $S C$ equilibrium of $\mathcal{E}$. Then there is $(p, q, y) \in P \times Q \times Y$ such that $\left(p, q, x^{*}, y\right)$ is a naïve complete equilibrium of $\mathcal{E}$.

Proof

Since $\left(p^{*}, q^{*}, x^{*}, y^{*}\right)$ is a naïve $\mathrm{SC}$ equilibrium, it holds that

$$
\left(x_{\cdot \mid s(t)}^{* h}, y_{\cdot \mid s(t)}^{* h}\right) \in{ }^{n} \bar{\delta}_{s(t)}^{h}\left(p_{\cdot \mid s(t)}^{*}, q_{\cdot \mid s(t)}^{*}, y_{s(t) \mid s(t-1)}^{* h}, e_{t+1, T \mid s(t)}^{h}\right) .
$$

For $s(t), \sigma(\tau)$ with $\tau>t$, we define $\mu_{\sigma(\tau) \mid s(t)}$ as the unique real number satisfying $q_{\sigma(\tau) \mid s(t)}^{*}=$ $\mu_{\sigma(\tau) \mid s(t)} p_{\sigma(\tau) \mid s(t)}^{*}$, see Theorem 4.3.

Next, we define $p_{s(t) \mid s(t)}=p_{s(t) \mid s(t)}^{*}, p_{\sigma(\tau) \mid s(t)}=p_{\sigma(\tau) \mid s(t)}^{*} \prod_{\tau^{\prime}=t+1}^{\tau} \mu_{\sigma\left(\tau^{\prime}\right) \mid s(t)}, q_{(\sigma(\tau), T \mid \sigma(\tau-1)) \mid s(t)}=$ $p_{\sigma(\tau), T \mid s(t)}$. Let $y_{(\sigma(\tau+1) \mid \sigma(\tau)) \mid s(t)}^{h}=y_{\sigma(\tau+1) \mid s(t)}^{* h}$ and $y_{(\sigma(\tau+2), T \mid \sigma(\tau)) \mid s(t)}^{h}=e_{\cdot \mid \sigma(\tau+2)}^{h}$ for every $\tau \geq t$ and every $\sigma$ with $\sigma(t)=s(t)$. Then

$$
\left(x_{\cdot \mid s(t)}^{* h}, y_{\cdot \mid s(t)}^{h}\right) \in{ }^{n} \gamma_{s(t)}^{h}\left(p_{\cdot \mid s(t)}, q_{\cdot \mid s(t)}, y_{s(t) \mid s(t-1)}^{* h}, e_{t+1, T \mid s(t)}^{h}\right) .
$$

Suppose that $\left(x_{\cdot \mid s(t)}^{* h}, y_{\cdot \mid s(t)}^{h}\right) \notin{ }^{n} \delta_{s(t)}^{h}\left(p_{\cdot \mid s(t)}, q_{\cdot \mid s(t)}, y_{s(t) \mid s(t-1)}^{* h}, e_{t+1, T \mid s(t)}^{h}\right)$. Then there must be $\left(x_{\cdot \mid s(t)}^{h}, \tilde{y}_{\cdot \mid s(t)}^{h}\right) \in{ }^{n} \gamma_{s(t)}^{h}\left(p_{\cdot \mid s(t)}, q_{\cdot \mid s(t)}, y_{s(t) \mid s(t-1)}^{* h}, e_{t+1, T \mid s(t)}^{h}\right)$ with $x_{\cdot \mid s(t)}^{h} \succ^{h, s(t)} x_{\cdot \mid s(t)}^{* h}$. As in the proof of Theorem 4.6 it follows that there is $\widehat{y}_{\cdot \mid s(t)}^{h}$ with

$$
\left(x_{\cdot \mid s(t)}^{h}, \widehat{y}_{\cdot \mid s(t)}^{h}\right) \in{ }^{n} \bar{\gamma}_{s(t)}^{h}\left(p_{\cdot \mid s(t)}^{*}, q_{\cdot \mid s(t)}^{*}, y_{s(t) \mid s(t-1)}^{* h}, e_{t+1, T \mid s(t)}^{h}\right) .
$$

This then contradicts the fact that $\left(x_{\cdot \mid s(t)}^{* h}, y_{\cdot \mid s(t)}^{* h}\right) \in{ }^{n} \bar{\delta}_{s(t)}^{h}\left(p_{\cdot \mid s(t)}^{*}, q_{\cdot \mid s(t)}^{*}, y_{s(t) \mid s(t-1)}^{* h}, e_{t+1, T \mid s(t)}^{h}\right)$. Q.E.D.

Surprisingly, there are naïve complete equilibria that are not allocationally equivalent to any naïve SC equilibrium. This is shown in the following example.

Example 5.9 Consider Example 4.5. We reinterpret the variables. There are four periods. In every period there is only one good. There is no uncertainty. The endowments are $e_{\cdot \mid 1}^{1}=(2,2,2,2)$ for household 1 and $e_{\cdot \mid 1}^{2}=(2,2,2,2)$ for household 2 .

Now consider the complete market structure. Let $p_{\cdot \mid 1}^{*}=(1 / 2,1 / 2,1 / 2,1 / 2), q_{(\cdot \mid 1) \mid 1}^{*}=$ $(1 / 2,1 / 2,1 / 2), q_{(\cdot \mid 2) \mid 1}^{*}=(1 / 2,1 / 2), q_{(\cdot \mid 3) \mid 1}^{*}=1 / 2, p_{\cdot \mid 2}^{*}=(1 / 2,1 / 2,1 / 2), q_{(\cdot \mid 2) \mid 2}^{*}=(1 / 2,1 / 2)$, $q_{(\cdot \mid 3) \mid 2}^{*}=1 / 2, p_{\cdot \mid 3}^{*}=(3 / 4,1 / 4), q_{(\cdot \mid 3) \mid 3}^{*}=1 / 4$. Consider the following allocation

$$
\begin{array}{ll}
x_{\cdot \mid 1}^{* 1}=(2,2,2,2) & x_{\cdot \mid 1}^{* 2}=(2,2,2,2) \\
x_{\cdot \mid 2}^{* 1}=(2,2,2) & x_{\cdot \mid 2}^{* 2}=(2,2,2) \\
x_{\cdot \mid 3}^{* 1}=(1,1) & x_{\cdot \mid 3}^{* 2}=(3,3) \\
x_{\cdot \mid 4}^{* 1}=1 & x_{\cdot \mid 4}^{* 2}=3
\end{array}
$$

achieved by the asset allocations

$$
\begin{array}{ll}
y_{(\cdot \mid 1) \mid 1}^{* 1}=(2,0,4) & y_{(\cdot \mid 1) \mid 1}^{* 2}=(2,4,0) \\
y_{(\cdot \mid 2) \mid 2}^{* 1}=(0,4) & y_{(\cdot \mid 2) \mid 2}^{* 2}=(4,0) \\
y_{(\cdot \mid 3) \mid 3}^{* 1}=1 & y_{(\cdot \mid 3) \mid 3}^{* 2}=3
\end{array}
$$


and

$$
\begin{array}{ll}
y_{(\cdot \mid 2) \mid 1}^{* 1}=(0,4) & y_{(\cdot \mid 2) \mid 1}^{* 2}=(4,0) \\
y_{(\cdot \mid 3) \mid 1}^{* 1}=2 & y_{(\cdot \mid 3) \mid 1}^{* 2}=2 \\
y_{(\cdot \mid 3) \mid 2}^{* 1}=2 & y_{(\cdot \mid 3) \mid 2}^{* 2}=2 .
\end{array}
$$

It can be verified that $\left(p^{*}, q^{*}, x^{*}, y^{*}\right)$ is a naïve complete equilibrium. In periods 1 and 2 , expected relative prices of the good in period 3 in terms of the good in period 4 is 1 and in period 3 this same relative price rises to 3 . Thus, households have incorrect expectations about prices.

Consider the case with sequentially complete markets. A naïve SC equilibrium with the same actual consumption does not exist. If household 1 would like to consume 2 units both in period 1 and in period 2, then that household would not buy or sell any assets in period 1 or period 2. Then, in period 3, starting with the initial endowments of 2 units of the good in each period, the only resulting equilibrium from then on is to consume these initial endowments. Thus, there are naïve complete equilibria that are not allocationally equivalent to any naïve SC equilibrium.

We already mentioned that there is no relation between AD equilibria on the one hand and naïve (sophisticated) SC or naïve (sophisticated) complete equilibria on the other hand when preferences are not time-consistent. Theorem 5.8 shows that every naïve SC equilibrium is allocationally equivalent to some complete equilibrium and Example 5.9 shows that the converse is not true. When we consider sophisticated behavior, however, sophisticated SC equilibria are allocationally equivalent to sophisticated complete equilibria and vice versa. This is shown in the following theorem.

Theorem 5.10 Let the economy $\mathcal{E}$ be locally non-satiated. Every sophisticated SC equilibrium of $\mathcal{E}$ is allocationally equivalent to some sophisticated complete equilibrium of $\mathcal{E}$ and vice versa.

Proof

Step 1

Consider $p \in P, q \in Q, \bar{q} \in \bar{Q}$, and, for every $s, \sigma$, for every $\tau, t$ with $\tau \geq t$ and $\sigma(t)=s(t)$, $\mu_{\sigma(\tau) \mid s(t)} \in \mathbb{R}$ such that

$$
\begin{aligned}
p_{s(\tau), T \mid s(t)} & =p_{\cdot \mid s(\tau)} \\
\bar{q}_{\sigma(\tau) \mid s(t)} & =\mu_{\sigma(\tau) \mid s(t)} p_{\sigma(\tau) \mid s(t)} \\
q_{(\sigma(\tau), T \mid \sigma(\tau-1)) \mid s(t)} & =\mu_{\sigma(\tau) \mid s(t)}\left(p_{\sigma(\tau) \mid s(t)}, q_{(\cdot \mid \sigma(\tau)) \mid s(t)}\right), \\
p_{\sigma(\tau) \mid s(t)} & \neq 0 .
\end{aligned}
$$

For every $s(\tau)$, for every $y_{\cdot \mid s(\tau-1)}^{h} \in Y_{\cdot \mid s(\tau-1)}^{h}$, for every $\bar{y}_{\cdot \mid s(\tau-1)}^{h} \in \bar{Y}_{\cdot \mid s(\tau-1)}^{h}$, we will prove that whenever household $h$ starts with the same income in both market structures, i.e.

$$
\begin{gathered}
p_{s(\tau) \mid s(\tau)} y_{(s(\tau) \mid s(\tau-1)) \mid s(\tau-1)}^{h}+\sum_{\sigma(\tau+1) \in s^{+}(\tau)} q_{(\sigma(\tau+1), T \mid s(\tau)) \mid s(\tau)} y_{(\sigma(\tau+1), T \mid s(\tau-1)) \mid s(\tau-1)}^{h}= \\
p_{s(\tau) \mid s(\tau)} \bar{y}_{s(\tau) \mid s(\tau-1)}^{h}+\bar{q}_{\cdot \mid s(\tau)} e_{\tau+1, T \mid s(\tau)}^{h},
\end{gathered}
$$


we have that:

$$
\left(x_{\cdot \mid s(\tau)}^{h}, y_{\cdot \mid s(\tau)}^{h}\right) \in{ }^{s} \gamma_{s(\tau)}^{h}\left(p_{\cdot \mid s(\tau)}, q \cdot \mid s(\tau), y_{(\cdot \mid s(\tau-1)) \mid s(\tau-1)}^{h}\right)
$$

implies that there is $\bar{y}_{\cdot \mid s(\tau)}^{h} \in \bar{Y}_{\cdot \mid s(\tau)}^{h}$ such that

$$
\left(x_{\cdot \mid s(\tau)}^{h}, \bar{y}_{\cdot \mid s(\tau)}^{h}\right) \in{ }^{s} \bar{\gamma}_{s(\tau)}^{h}\left(p_{\cdot \mid s(\tau)}, \bar{q}_{\cdot \mid s(\tau)}, \bar{y}_{s(\tau) \mid s(\tau-1)}^{h}, e_{\tau+1, T \mid s(\tau)}^{h}\right)
$$

and vice versa. We give a proof by induction.

[Step 1a]

First of all, it is obvious that for every $\bar{y}_{s(T) \mid s(T-1)}^{h}, y_{s(T) \mid s(T-1)}^{h}$ with

$$
p_{\cdot \mid s(T)} \bar{y}_{s(T) \mid s(T-1)}^{h}=p_{\cdot \mid s(T)} y_{s(T) \mid s(T-1)}^{h}
$$

it holds that

$$
{ }^{s} \bar{\gamma}_{s(T)}^{h}\left(p_{\cdot \mid s(T)}, \bar{y}_{s(T) \mid s(T-1)}^{h}\right)={ }^{s} \gamma_{s(T)}^{h}\left(p_{\cdot \mid s(T)}, y_{s(T) \mid s(T-1)}^{h}\right) .
$$

[Step 1b]

Assume the result is true for every $s(\tau)$, where $\tau>t$.

Now assume that, in state $s(t)$, household $h$ starts with the same income in both market structures, i.e.

$$
\begin{aligned}
p_{s(t) \mid s(t)} y_{(s(t) \mid s(t-1)) \mid s(t-1)}^{h}+ & \sum_{\sigma(t+1) \in s^{+}(t)} q_{(\sigma(t+1), T \mid s(t)) \mid s(t)} y_{(\sigma(t+1), T \mid s(t-1)) \mid s(t-1)}^{h}= \\
& p_{s(t) \mid s(t)} \bar{y}_{s(t) \mid s(t-1)}^{h}+\bar{q}_{\cdot \mid s(t)} e_{t+1, T \mid s(t)}^{h} .
\end{aligned}
$$

We first prove one direction of our result.

$(\Rightarrow \quad$ Let

$$
\left(x_{\cdot \mid s(t)}^{h}, y_{\cdot \mid s(t)}^{h}\right) \in{ }^{s} \gamma_{s(t)}^{h}\left(p_{\cdot \mid s(t)}, q_{\cdot \mid s(t)}, y_{(\cdot \mid s(t-1)) \mid s(t-1)}^{h}\right) .
$$

We define $\bar{y}_{\cdot \mid s(t)}^{h}$ recursively as follows. For every $\sigma(\tau), s(t)$ there is a $l_{\sigma(\tau) \mid s(t)}$ with $p_{\sigma(\tau), l_{\sigma(\tau) \mid s(t)} \mid s(t)} \neq$ 0 . Start by setting, for every $h \in H$,

$$
\bar{y}_{\sigma(T), l_{\sigma(T) \mid s(t)} \mid s(t)}^{h}=\frac{p_{\sigma(T) \mid s(t)}\left(x_{\sigma(T) \mid s(t)}^{h}-e_{\sigma(T) \mid s(t)}^{h}\right)}{p_{\sigma(T), l_{\sigma(T) \mid s(t)} \mid s(t)}}+e_{\sigma(T), l_{\sigma(T) \mid s(t)} \mid s(t)}^{h},
$$

and $\bar{y}_{\sigma(T), l \mid s(t)}^{h}=e_{\sigma(T), l \mid s(t)}^{h}$ for every $l \neq l_{\sigma(T) \mid s(t)}$. Then, continue by setting, for every $h \in H$, $t<\tau<T, \sigma \in S$ with $\sigma(t)=s(t)$,

$$
\begin{aligned}
\bar{y}_{\sigma(\tau), l_{\sigma(\tau) \mid s(t)}^{h} \mid s(t)} & =\frac{p_{\sigma(\tau) \mid s(t)}\left(x_{\sigma(\tau) \mid s(t)}^{h}-e_{\sigma(\tau) \mid s(t)}^{h}\right)}{p_{\sigma(\tau), l_{\sigma(\tau) \mid s(t)} \mid s(t)}}+e_{\sigma(\tau), l_{\sigma(\tau) \mid s(t)} \mid s(t)}^{h} \\
& +\frac{\sum_{\sigma(\tau+1) \in \sigma^{+}(\tau)}\left(\bar{q}_{\sigma(\tau+1) \mid s(t)} \bar{y}_{\sigma(\tau+1) \mid s(t)}^{h}-\bar{q}_{\sigma(\tau+1) \mid s(t)} e_{\sigma(\tau+1) \mid s(t)}^{h}\right)}{p_{\sigma(\tau), l_{\sigma(\tau) \mid s(t)} \mid s(t)}}
\end{aligned}
$$


and $y_{\sigma(\tau), l \mid s(t)}^{* h}=e_{\sigma(\tau), l \mid s(t)}^{h}$ for every $l \neq l_{\sigma(\tau) \mid s(t)}$.

Then,

$$
\begin{gathered}
p_{s(t+1) \mid s(t+1)} \bar{y}_{s(t+1) \mid s(t)}^{h}+\sum_{\sigma(t+2) \in s^{+}(t+1)} \bar{q}_{\sigma(t+2), T \mid s(t)} e_{\sigma(t+2), T \mid s(t)}^{h}= \\
p_{s(t+1) \mid s(t+1)} x_{s(t+1) \mid s(t)}^{h}+\sum_{\sigma(t+2) \in s^{+}(t+1)} \bar{q}_{\sigma(t+2), T \mid s(t)} x_{\sigma(t+2), T \mid s(t)}^{h}
\end{gathered}
$$

and

$$
\begin{array}{r}
p_{s(t+1) \mid s(t+1)} y_{(s(t+1) \mid s(t)) \mid s(t)}^{h}+\sum_{\sigma(t+2) \in s^{+}(t+1)} q_{(\sigma(t+2), T \mid s(t+1)) \mid s(t+1)} y_{(\sigma(t+2), T \mid s(t)) \mid s(t)}^{h}= \\
p_{s(t+1) \mid s(t+1)} x_{s(t+1) \mid s(t)}^{h}+\sum_{\sigma(t+2) \in s^{+}(t+1)} q_{(\sigma(t+2), T \mid s(t+1)) \mid s(t+1)} x_{\sigma(t+2), T \mid s(t)}^{h}
\end{array}
$$

so it follows that

$$
\begin{aligned}
p_{s(t+1) \mid s(t+1)} y_{(s(t+1) \mid s(t)) \mid s(t)}^{h}+ & \sum_{\sigma(t+2) \in s^{+}(t+1)} q_{(\sigma(t+2), T \mid s(t+1)) \mid s(t+1)} y_{(\sigma(t+2), T \mid s(t)) \mid s(t)}^{h}= \\
& p_{s(t+1) \mid s(t+1)} \bar{y}_{s(t+1) \mid s(t)}^{h}+\sum_{\sigma(t+2) \in s^{+}(t+1)} \bar{q}_{\sigma(t+2), T \mid s(t)} e_{\sigma(t+2), T \mid s(t)}^{h} .
\end{aligned}
$$

Using our induction hypothesis, we obtain easily that

$$
\left(x_{s(t+1), T \mid s(t)}^{h}, \bar{y}_{s^{+}(t+1), T \mid s(t)}^{h}\right) \in s \bar{\delta}_{s(t+1)}^{h}\left(p_{s(t+1), T \mid s(t)}, \bar{q}_{s^{+}(t+1), T \mid s(t)}, \bar{y}_{s(t+1) \mid s(t)}^{h}, e_{t+2, T \mid s(t+1)}^{h}\right) .
$$

Since the income of household $h$ in state $s(t)$ is the same under both market structures, it follows that

$$
\left(x_{\cdot \mid s(t)}^{h}, \bar{y}_{\cdot \mid s(t)}^{h}\right) \in{ }^{s} \bar{\gamma}_{s(t)}^{h}\left(p_{\cdot \mid s(t)}, \bar{q}_{\cdot \mid s(t)}, \bar{y}_{s(t) \mid s(t-1)}^{h}, e_{t+1, T \mid s(t)}^{h}\right)
$$

$(\Leftarrow) \quad$ The other direction of our result is straightforward, when following the approach used in Theorem 5.8

Step 2

$\left.\overline{\text { Let }\left(p^{*}\right.}, q^{*}, x^{*}, y^{*}\right)$ be a sophisticated SC equilibrium. By local non-satiation we know that for all $\sigma(\tau), p_{\sigma(\tau) \mid 1}^{*} \neq 0$. By Theorem 4.4 there is $\mu_{\sigma(\tau) \mid 1} \in \mathbb{R}$ such that

$$
q_{\sigma(\tau) \mid 1}^{*}=\mu_{\sigma(\tau) \mid 1} p_{\sigma(\tau) \mid 1}^{*}
$$

We define $q \in Q$ as follows.

For every $\sigma(T)$,

$$
q_{(\sigma(T), T \mid \sigma(T-1)) \mid 1}=\mu_{\sigma(T) \mid 1}\left(p_{\sigma(T) \mid 1}^{*}\right) .
$$


Next, recursively, for $\tau$ descending from $T-1$ to 2 , for every $\sigma(\tau)$,

$$
q_{(\sigma(\tau), T \mid \sigma(\tau-1)) \mid 1}=\mu_{\sigma(\tau) \mid 1}\left(p_{\sigma(\tau) \mid 1}^{*}, q_{(\cdot \mid \sigma(\tau)) \mid 1}\right) .
$$

Finally, for every $s, \sigma$, for every $t, \tau$ with $\tau \geq t$ and $\sigma(t)=s(t)$,

$$
q_{(\cdot \mid \sigma(\tau)) \mid s(t)}=q_{(\cdot \mid \sigma(\tau)) \mid 1}
$$

From the previous analysis it follows that there is a $y$ such that $\left(p^{*}, q, x^{*}, y\right)$ is a sophisticated complete equilibrium.

Step 3

$\left.\overline{\text { Let }\left(p^{*}\right.}, q^{*}, x^{*}, y^{*}\right)$ be a sophisticated complete equilibrium. Consider any $s, \sigma$, any $t, \tau$ with $\tau \geq t$ and $\sigma(t)=s(t)$. By local non-satiation we know that $p_{\sigma(\tau) \mid s(t)}^{*} \neq 0$. We define

$$
\bar{q}_{\sigma(\tau) \mid s(t)}=q_{(\sigma(\tau) \mid \sigma(\tau-1)) \mid s(t)}^{*}
$$

From the previous analysis it follows that there is $y \in \bar{Y}$ such that $\left(p^{*}, \bar{q}, x^{*}, y\right)$ is a sophisticated SC equilibrium.

Q.E.D.

\section{Conclusion}

Arrow (1953) showed that every Pareto optimal allocation in an Arrow-Debreu economy can be achieved both by Arrow-Debreu markets and a particular sequentially complete market setting where first securities are traded and then spot markets are opened. Debreu (1959) claims that, when all contingent commodities can be traded in the first period and preferences of households do not change over time, there is no need for markets to reopen in later periods.

In this paper we allow preferences to be time-inconsistent and we consider three market structures. In this richer environment, we reconsider the results of Arrow (1953) and Debreu (1959).

We first consider the Arrow-Debreu setting where all contingent commodities can be traded in the first period and where markets are not reopened in later periods. Next, we consider sequentially complete markets where goods on the spot markets and all one-period ahead contingent commodities can be traded in every state. Finally, we consider complete markets where all contingent commodities can be traded in every state.

Following Pollak (1968) we make a distinction between naïve and sophisticated behavior. Naïve households do not realize that their preferences change over time, whereas sophisticated ones do. The latter will only make plans that they expect to carry out in the future.

For sophisticated households, we show that sequentially complete markets are allocationally equivalent to complete markets. For naïve households, every naïve sequentially complete equilibrium is allocationally equivalent to some naïve complete equilibrium, but the converse does not hold, even if we assume that preferences are time-consistent. 
When preferences are time-consistent and households are naïve, every equilibrium in the Arrow-Debreu setting is allocationally equivalent to some naïve sequentially complete equilibrium and to some naïve complete equilibrium. When preferences are time-consistent and, in addition, households are sophisticated all three market market structures are allocationally equivalent.

Sophisticated behavior requires a lot from households, however. A more reasonable type of behavior would be "somewhat" sophisticated. Loewenstein, O'Donoghue, and Rabin (2003) assume that households do make plans that they expect to stick to. However, they assume that households mispredict their future utility function and thus will want to reconsider their plans in the future. Here, we could model these households as having the same budget constraints as sophisticated households, but with different actual utility functions in later periods. For these households, it cannot be expected that they have perfect foresight of prices and thus, the results for the naïve households in this paper would apply. As soon as we allow at least one household not to be completely sophisticated, our paper shows that the degree of completeness matters.

\section{References}

Arrow, K.J., and G. Debreu (1954), "Existence of an Equilibrium for a Competitive Economy," Econometrica, 22, 265-290.

Arrow, K.J. (1953), "Le Rôle des Valeurs Boursières pour la Répartition la Meilleure des Risques," in Econométrie, Colloques Internationaux du Centre National de la Recherce Scientifique, 40, 41-47; English version: "The Role of Securities in the Optimal Allocation of Risk-Bearing," Review of Economic Studies, 31, 91-96.

Chattopadhyay, S., and P. Gottardi (1999), "Stochastic OLG Models, Market Structure, and Optimality," Journal of Economic Theory, 89, 21-67.

Debreu, G. (1959), Theory of Value, Wiley, New York.

Donaldson, J.B., and L. Selden (1981), "Arrow-Debreu Preferences and the Reopening of Contingent Claims Markets," Economics Letters, 8, 209-216.

Drèze, J.H., and P.J.J. Herings (2003), "Sequentially Complete Markets Remain Incomplete," METEOR Research Memorandum 03/44, Maastricht University, pp. 1-13.

Haller, H. (1990), "Non-Market Reopening, Time-Consistent Plans and the Structure of Intertemporal Preferences," Economics Letters, 32, 1-5.

Herings, P.J.J.., and K.I.M. Rohde (2004), "Time-inconsistent Preferences in a General Equilibrium Model," METEOR Research Memorandum 04/17, Maastricht University, pp. 
$1-41$.

Loewenstein, G., T. O'Donoghue, and M. Rabin (2003), "Projection Bias in Predicting Future Utility," Quarterly Journal of Economics, 118, 1209-1248.

Luttmer, E.G.J., and T. Mariotti (2003), "Subjective Discounting in an Exchange Economy," Journal of Political Economy, 111, 1-30.

Pollak, R.A. (1968), "Consistent Planning," Review of Economic Studies, 35, 201-208.

Svensson, L.E.O. (1976), "Sequences of Temporary Equilibria, Stationary Point Expectations and Pareto-Efficiency," Journal of Economic Theory, 13, 169-183. 